\title{
MEASUREMENTS OF EXTREME ULTRA-VIOLET SOLAR RADIATION USING A FILTER METHOD
}

\author{
By W. W. Coblentz and R. Stair
}

\section{ABSTRACT}

In this paper quantitative measurements are given of the component of ultraviolet solar radiation of wave lengths less than $313 \mathrm{in} \mu$.

The method used is a type of filter radiometry, emploving a nonselective thermocouple receiver, permanently covered with a filter that is highly transparent to short wave length ultra-violet radiation and practically opaque to the visible and the infra-red rays. By temporarily placing in the path of the rays first one, then two screens of barium flint glass which is opaque to wave lengths less than $313 \mathrm{~m} \mu$, the ultra-violet of wave lengths less than $313 \mathrm{~m} \mu$ is evaluated by exclusion from the total ultra-violet measured.

Measurements are given of the incident ultra-violet solar radiation on the clearest days, as affected by atmospheric pollution, altitude of the station, humidity, time of the day, and season of the year. These data are discussed and correlated with some of the measurements and calculations made by others.

\section{CONTENTS}

I. Introduction ...

II. Method of observation. 953

III. Reduction of data_...

IV. Discussion of data _....... 964

1. Effect of atmospheric pollution... 964

2. Effect of altitude

3. Effect of humidity on scattering _........ 967

4. Daily variation in ultra-violet radiation _......... 969

5. Seasonal variation in ultra-violet radiation........... 970

6. Ultra-violet in sky radiation

7. Comparison of data............ 972

V. Summary and acknowledgments.............. 974

VI. References ............ 975

\section{INTRODUCTIOIN}

The present communication is a further contribution to the general subject of instruments and methods of radiometry, that has been under investigation for some years (1). ${ }^{1}$ The particular problem considered, which is still under investigation, is the quantitative measurement of short wave length ultra-violet solar radiation.

The method of observation is by means of a nonselective radiometer, permanently covered with a filter that is highly transparent to the ultra-violet radiation of wave lengths less than $313 \mathrm{~m} \mu$ (millimicrons, $3130 \mathrm{~A}$ ) and opaque to practically all of the visible and the infra-red rays. Since this filter transmits ultra-violet of wave lengths longer than $313 \mathrm{~m} \mu$, the component of wave length less than $313 \mathrm{m \mu}$, is evaluated by temporarily placing in the path of the incident rays, first one, then two screens of barium flint glass, which exclude these rays from the total ultra-violet measured. In this sense, it is an exclusion method of filter radiometry.

1 Figures in parenthesis here and throughout the text indicate references glven in tbe Bibllography at the end of this paper. 
A pressing question of interest and far-reaching importance is a reliable method for evaluating the amount of ultra-violet radiation in sunlight of wave lengths less than about $313 \mathrm{~m} \mu$, which varies with the altitude and latitude of the station, the time of the day, the season of the year, cloudiness, and with the countless variations in atmospheric pollution by dust, smoke, etc., that are encountered in different localities.

This subject was discussed in a previous paper (2) in connection with the transparency of special window glasses for transmitting the short wave length ultra-violet solar radiation, of wave lengths less than about $313 \mathrm{~m} \mu$, recognized by biologists as having a specific healing: value, particularly in the prevention and cure of rickets.

From the preliminary observations given in the preceding paper (2), it was evident that in order to be of value, measurements obtained by this method, should be made only during cloudless intervals. Such a procedure is not objectionable, and, since no other methods equally promising were available at that time it was decided to give the filter method a trial, extending over an annual cycle in order to include winter and summer weather, at Washington. Measurements were made also at two stations at Flagstaff, Ariz., in order to determine the effect of altitude and humidity.

The data thus obtained contain some interesting information that will be discussed on a subsequent page. In a future communication it is proposed to give similar data obtained by a modification of Method III, previously described (2) in which the thermocouple was covered with only a $1 \mathrm{~cm}$ cell of water (with quartz windows) and the ultra-violet radiation of wave lengths less than $313 \mathrm{~m} \mu$ was excluded by means of a barium flint glass screen, as used in the present investigation.

The modification now under investigation consists in exposing both thermocouple receivers ("hot" and "cold," adjusted radiometrically closer than 1 per cent) to the sun, under which conditions practically no galvanometer deflection is obtained. By covering one thermocouple with the barium flint glass screen and the other receiver with a plate of Corex A glass or quartz (to compensate for reflection of radiation at 365 to $1,400 \mathrm{~m} \mu$ ) the galvanometer deflection, obtained on the simultaneous exposure of the two receivers to the sun, is a measure of the ultra-violet excluded by the flint glass filter. By interchanging the screens, differences in the two thermocouple receivers are eliminated.

The chief difficulty lies in obtaining two screens (one opaque to the ultra-violet and the other transparent to the ultra-violet, of wave lengths less than $313 \mathrm{~m} \mu$ ) having exactly the same transmission for wave lengths beginning at about $365 \mathrm{~m} \mu$ and extending to about $1,400 \mathrm{~m} \mu$. Owing to the large amount of radiation in this spectral region, slight differences in the transmission may cause errors in the radiation measurements that are greater than the ultra-violet component that it is desired to measure.

By covering both receivers with a yellow, Noviol C, glass, differences in transparency of the barium flint glass and the Corex $A$ or quartz screens, between 365 and $1,400 \mathrm{~m} \mu$, are determined and deducted from the ultra-violet at wave lengths less than $365 \mathrm{~m} \mu$ excluded by the barium flint glass filter.

The energy value of the ultra-violet shut out by the filter may be determined directly by calibration of the thermocouple receiver 
against a standard of radiation as in the previous measurements (2), or from the total solar intensity, $Q$ (and the reduction factor $F$ ) as described in the present paper. The results obtained by this new method are in good agreement with the data given in the present paper, from which it appears that reliable results may be obtained by using only one barium flint glass screen. It is a good check on the present data by a widely different method.

If desired, this device can be connected with a recording apparatus, thus obtaining a continuous record as used by Pettit (9). However, owing to the large amount of radiation incident upon the water cell $\left(1.2 \mathrm{~g}\right.$ cal. $/ \mathrm{cm}^{2} / \mathrm{min}$. as compared with about 8 per cent of this value when using the herein described ultra-violet filter) care must bo taken to avoid heating of the cell. In this respect the present method, in which the ultra-violet filter transmits over 80 per cent of the short wave length ultra-violet (without distortion of the shape of the solar spectral energy curve of wave lengths less than $350 \mathrm{~m} \mu$ ) but transmits only 8 per cent of the total solar intensity, $Q$, seems preferable. The ultra-violet filter is to be used also in the new method when it is not being used to check the present method.

With reference to the data presented in the present paper the measurements obtained in Washington are of particular interest in showing the seasonal variation in ultra-violet radiation that may be expected at a sea-level station which is not subjected to atmospheric pollution by factory smoke, but has to contend mainly with pollution by the combustion products from automobiles, and with the increasing numbers of incinerators and oil burners, in office buildings, apartment houses, and private dwellings, which emit not only smoke but also atomized particles of oil.

In this connection it is relevant to anticipate the discussion of the present observations by citing the measurements of October 8, 1929, when the northwest section of the city of Washington was enveloped in a rather dense low-lying stratum of finely divided particles that absorbed the short wave length ultra-violet rays. The atmosphere was cloudless and windstill, and the stratum of bluish haze was easily discernible, extending to a height of 500 to 1,000 feet. As the sun mounted higher in the sky, it shone through a decreasing thickness of this stratum, and the amount of ultra-violet transmitted was greatly increased. For example, the ultra-violet component measured at $9.15 \mathrm{a}$. $\mathrm{m}$. was about two times the valuo measured 15 minutes earlier when the sun's rays traversed a longer path of smoke.

\section{METHOD OF OBSERVATION}

As outlined in a previous paper (2) the method used consists essentially in isolating the ultra-violet radiation by a purple glass filter, Corning G986A, which is highly transparent to ultra-violet radiation of wave lengths between 250 and $440 \mathrm{~m} \mu(t r=\$ 0$ to $\$ 1$ per cent between 310 and $360 \mathrm{~m} \mu$ ) and measuring the intensity of the transmitted rays by means of a thermocouple (of copper-constantan, in air) having a receiving surface about $2.8 \mathrm{~mm}$ in diameter. A water cell of fused quartz, $1 \mathrm{~cm}$ in thickness, combined with tho purple glass filter, G986A, absorbs all the infra-red rays of ware lengths longer than about $1,400 \mathrm{~m} \mu$. A correction is easily obtained for the small amount of infra-red rays, of wave lengths 700 to 1,400 $\mathrm{m} \mu$, transmitted by the glass filter. 
The ultra-violet solar radiation of wave lengths 290 to $313 \mathrm{~m} \mu$ is obtained by exclusion, by inserting a plate of barium flint glass (G669, thickness $=3.05 \mathrm{~mm}$ ) having a transmission of 0.5 per cent at $313 \mathrm{~m} \mu, 49$ to 50 per cent at $334 \mathrm{~m} \mu, 85$ per cent at $365 \mathrm{~m} \mu$, and 90.7 per cent or a trifle higher throughout the visible and infra-red to $1,400 \mathrm{~m} \mu$ and longer wave lengths. The long wave length limit of the ultra-violet cut-off is taken at (and including) $313 \mathrm{~m} \mu$ instead of $310 \mathrm{~m} \mu$ because it is a definite and easily obtained emission line (of the mercury arc spectrum) and, also, because biological action, at least in preventing rickets, terminates practically at this wave length (6).

The complete mounting, on its improvised polar axis, is shown in figure 1. The shutter, $S$, is shown opened to the left. The small aluminum disk, $D$, was used to shield the thermocouple receiver from the direct rays of the sun when measurements were made of the sky radiation. The notches in the diaphragmed opening show the compartments for the water cell and the filters.

The diaphragmed opening (19) was purposely constructed to admit radiation from a solid angle of $45^{\circ}$ to the sky, which, by scattering, contributes ( 6 to 10 per cent or more, Table 2) ultra-violet radiation useful for therapeutic purposes. The whole metal box containing the thermocouple and diaphragmed top was wrapped in white cotton batting in order to provide a highly reflecting, thermally nonconducting medium, to reduce the heating on exposure to sunlight.

The experimental procedure consisted in exposing the copperconstantan thermosouple (permanently covered with the $1 \mathrm{~cm}$ cell of water and the purple glass, G986A) to the sun and noting the decrease in the galvanometer deflections when increasing amounts of ultra-violet radiation were shut out $(a)$ by one filter, then $(b)$ by two filters of barium flint glass, G669. The combination of two samples of glass is used to measure the ultra-violet spectral energy distribution in the region of 313 to $365 \mathrm{~m} \mu$, and gives some interesting information regarding the variation in energy in this spectral region, which variation is not necessarily in coincidence with the variation in ultra-violet of wave lengths less than $313 \mathrm{~m} \mu$ shut out by the first screen.

As a matter of information it is relevant to add that in the Washington measurements an ironclad Thomson galvanometer was generally used; also a high sensitivity d'Arsonval galvanometer having a single swing of about seven seconds. At the Lowell Observatory, Flagstaff, Ariz. (altitude 7,250 feet), and on the San Francisco Peaks (near Flagstaff, altitude 10,500 feet) the greater solar intensity warmed the water cell so rapidly (thus affecting the thermocouple) that it was necessary to use a d'Arsonval galvanometer having a single swing of two to three seconds.

An Angström pyrheliometer was used to measure the solar radiation intensities at the Flagstaff stations. For the Washington comparisons the solar radiation intensities were kindly supplied by I. F. Hand, of the United States Weather Bureau.

It is to be noted that measurements were made only on apparently cloudless days, or parts of days, when sky conditions remained uniform for an hour or more. By making 20 to 40 readings, in Washington, and about 10 readings at the Flagstaff stations, it was possible to attain an accuracy of 1 per cent or greater. The great variations 
B. S. Journal of Research, RP318

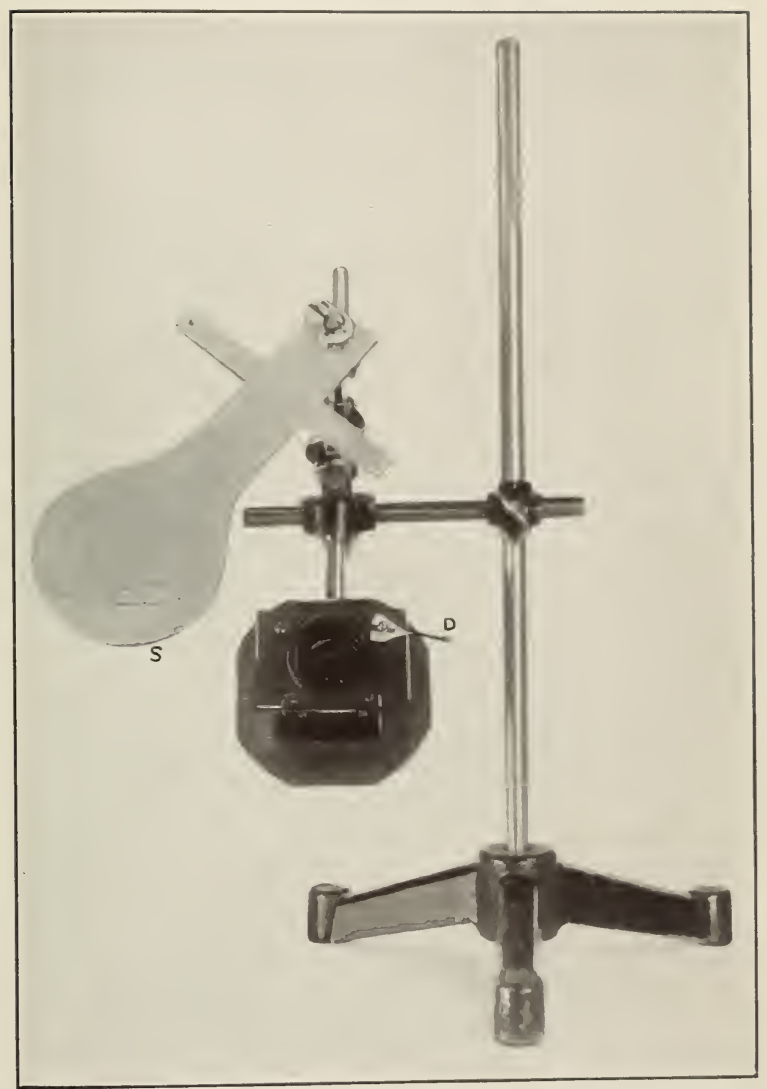

Figure 1. Front view of ultra-violet radiometer

The notches in the diaphragmed opening show the compartments for the filters. 
in the measurements are, therefore, to be ascribed to atmospheric conditions, which (at sea level) are never stationary as regards transmission of ultra-violet radiation, however uniform the sky may appear to the eye.

This is easily observed with a photo-electric cell that is sensitive only to ultra-violet radiation of wave lengths less than $313 m \mu$. From their experience with quick-acting photo-electric cells which respond to slight changes in atmospheric transparency, the writers have found it extremely difficult to obtain accurate measurements of the ultra-violet in direct sunlight. This unsteadiness is scarcely noticeable when measuring, with a photo-electric cell, the ultraviolet radiation from wide expanses of the sky. The reasons for these great variations become apparent in going from a low to a high elevation, where the observer becomes aware of the narrow range that obtains in the equilibrium conditions of the temperature and the pressure of the air (at least at certain seasons of the year) as indicated by the sudden appearance, and equally sudden disappearance, of small diaphanous clouds in an otherwise cloudless sky. The temporary. condensation of much larger masses of water vapor is necessary in order to be perceptible from a sea-level station. Their presence may be accentuated by looking through a sage green or yellowish green glass.

Owing to the presence of trees that shadowed the laboratory at the noon hour and part of the afternoon in the winter, the observations at Washington are incomplete. However, since there is but little change in air mass at the noon hour in winter, no attempt was made to change the observing station during this investigation.

\section{REDUCTION OF DATA}

Using this method of making the measurements it is an easy matter to determine, in arbitrary units, the amount of biologically active ultra-violet radiation shut out by common window glass, and note the variation in amount with change in season, atnospheric pollution, etc.

TABLE 1.-Ultra-violet solar radiation intensity, $u . v . Q$. of wave lengths less than $313 \mathrm{m \mu}$, also the total solar radiation intensity, $Q$, in $\mathrm{g} \mathrm{cal.} / \mathrm{cm}^{2} / \mathrm{min}$., for different air masses, m, at Flagstaff, Ariz. (altitude 7,250 feet), and on the San Francisco Peaks (altitude 10,500 feet)

[The time is Mountain Standard (M. S. T.)]

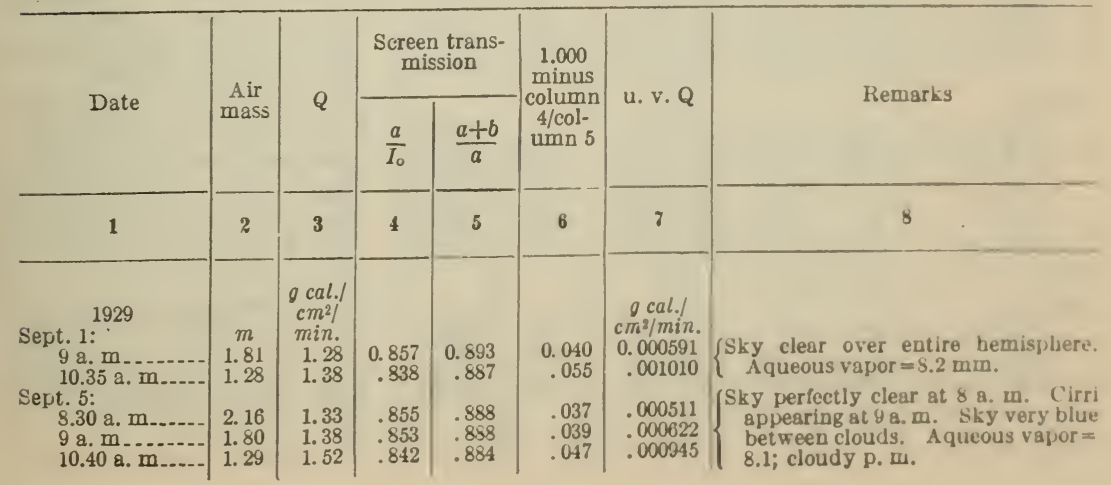


TABLE 1.-Ultra-violet solar radiation intensity, $u . v . Q . \cdot$ of wave lengths less than

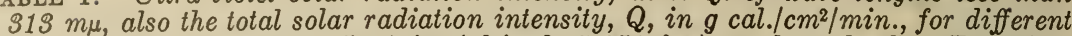
air masses, m, at Flagstaff, Ariz. (altitude 7,250 feet), and on the San Francisco Peaks (altitude 10,500 feet)-Continued.

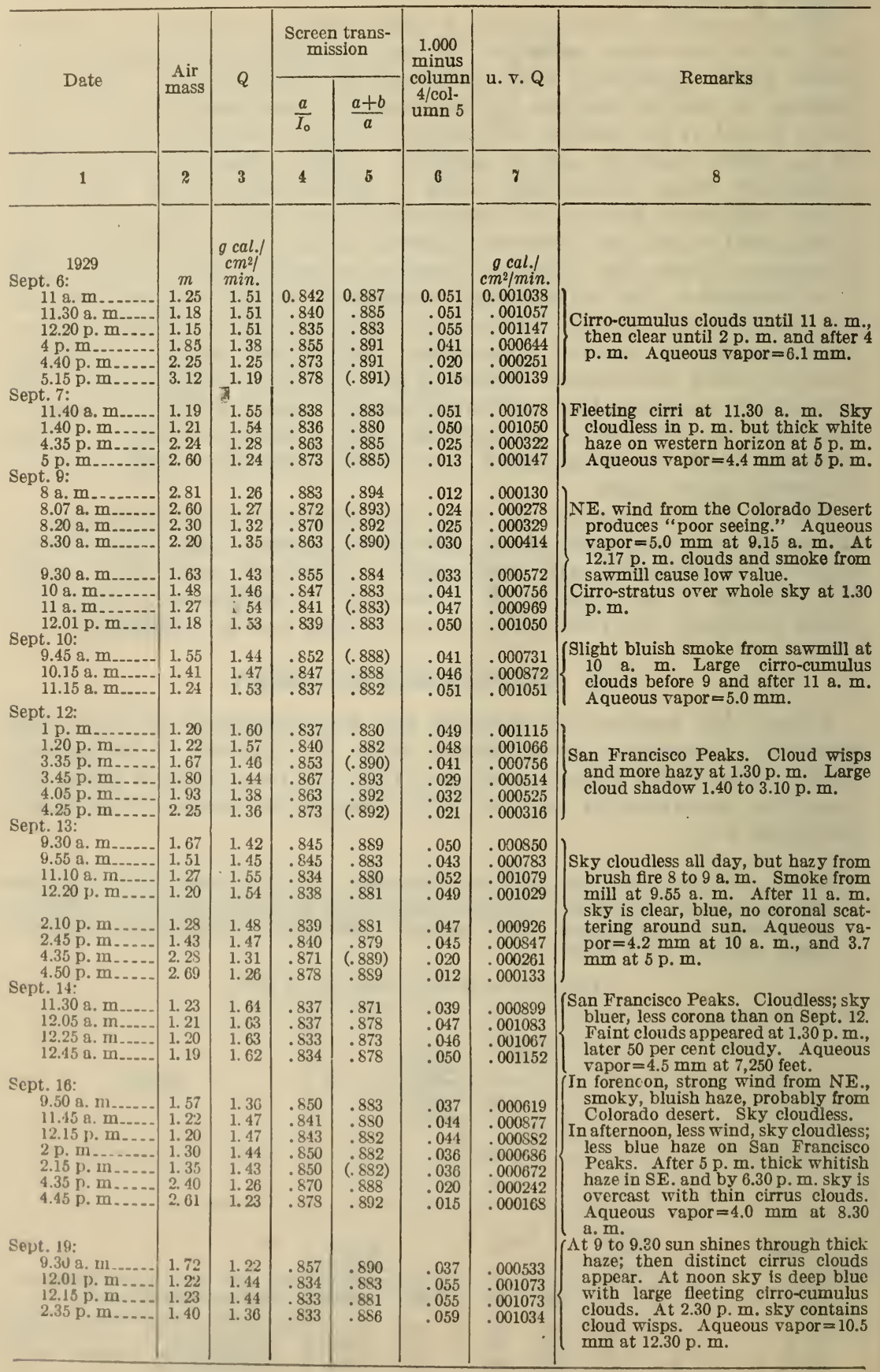


TABLE 2.-Ultra-violet solar radiation intensity, $u . v . Q$, of wave lengths less than $313 \mathrm{m \mu}$, also the total solar radiation intensity, $Q$, in $g \mathrm{cal} . / \mathrm{cm}^{2} / \mathrm{min}$., for different air masses, $m$, at Washington, D. C. (altitude $\$ 50$ feet.)

[The time is eastern standard (E. S. T.). In column 5 the interpolated ralues are in parentheses]

\begin{tabular}{|c|c|c|c|c|c|c|c|}
\hline \multirow{2}{*}{ Date } & \multirow{2}{*}{ Air } & \multirow{2}{*}{$Q$} & \multicolumn{2}{|c|}{$\begin{array}{l}\text { Screen trans- } \\
\text { mission }\end{array}$} & \multirow{2}{*}{$\begin{array}{c}1.000 \\
\text { minus } \\
\text { column } \\
4 / \text { col- } \\
\text { umn } 5\end{array}$} & \multirow{2}{*}{ u. o. Q } & \multirow{2}{*}{ Rezาarks } \\
\hline & & & $\frac{a}{I_{0}}$ & $\frac{a+b}{a}$ & & & \\
\hline 1 & 2 & 3 & 4 & 5 & 6 & 7 & 8 \\
\hline $\begin{array}{l}\text { Oct. } 8: \\
9 \mathrm{a} . \mathrm{m} \\
9.15 \mathrm{a} \cdot \mathrm{m} \\
10 \mathrm{a} . \mathrm{m} \\
11 \mathrm{a} \cdot \mathrm{m} \\
12 \mathrm{a} \cdot \mathrm{m} \\
\text { Oct. } 9:\end{array}$ & $\begin{array}{l}m \\
2.01 \\
1.94 \\
1.53 \\
1.46 \\
1.41\end{array}$ & $\begin{array}{l}g \text { cal./ } \\
\mathrm{cm}^{2} / \\
\min . \\
0.92 \\
.99 \\
1.15 \\
1.24 \\
1.28\end{array}$ & $\begin{array}{l}0.880 \\
.865 \\
.868 \\
.863 \\
.861\end{array}$ & $\begin{array}{l}0.898 \\
(.893) \\
.893 \\
.895 \\
(.894)\end{array}$ & $\begin{array}{l}0.021 \\
.031 \\
.028 \\
.036 \\
.037\end{array}$ & $\begin{array}{c}g \text { cal.l } \\
\mathrm{cm}^{2} / \mathrm{min} . \\
0.000175 \\
.000285 \\
.000350 \\
.000504 \\
.000547\end{array}$ & $\begin{array}{l}\text { Sky cloudless all day. Low stratum } \\
\text { of bluo haze at } 8 \text { to } 10 \mathrm{a} . \mathrm{m} \text {. }\end{array}$ \\
\hline $\begin{array}{l}\text { Oct. } 9: \\
\quad 9 \text { a. m } \\
\quad 9.15 \mathrm{a} . \mathrm{m} \\
\quad 12.01 \mathrm{p} . \mathrm{m} \\
\text { Oct. } 18 \text { : }\end{array}$ & $\begin{array}{l}2.02 \\
1.96 \\
1.43\end{array}$ & $\begin{array}{r}.69 \\
.78 \\
1.11\end{array}$ & $\begin{array}{l}.883 \\
.880 \\
.884\end{array}$ & $\begin{array}{l}(.895) \\
(.895) \\
.902\end{array}$ & $\begin{array}{l}.013 \\
.017 \\
.020\end{array}$ & $\begin{array}{l}.000080 \\
.000123 \\
.000253\end{array}$ & $\begin{array}{l}\text { Morning clear. Clouds between } \\
9.30 \text { and } 11 \mathrm{a} . \mathrm{m} \text {. Thick haze at } \\
\text { noon. }\end{array}$ \\
\hline 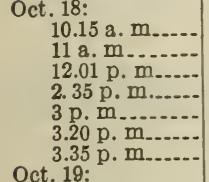 & $\begin{array}{l}1.73 \\
1.54 \\
1.48 \\
2.00 \\
2.40 \\
2.60 \\
3.00\end{array}$ & $\begin{array}{l}1.27 \\
1.30 \\
1.35 \\
1.12 \\
1.02 \\
1.00 \\
.92\end{array}$ & $\begin{array}{l}.863 \\
.858 \\
.863 \\
.867 \\
.870 \\
.880 \\
.888\end{array}$ & $\begin{array}{l}.898 \\
(.895) \\
.893 \\
(.893) \\
(.895) \\
(895) \\
(898)\end{array}$ & $\begin{array}{l}.039 \\
.041 \\
.033 \\
.029 \\
.028 \\
.017 \\
.011\end{array}$ & $\begin{array}{l}.000502 \\
.000575 \\
.000496 \\
.000294 \\
.000220 \\
.000120 \\
.000058\end{array}$ & Clear. Cloudless after trost. \\
\hline $\begin{array}{l}\text { Oct. 19: } \\
11 \mathrm{a} . \mathrm{m} \\
11.45 \mathrm{a} . \mathrm{m}\end{array}$ & $\begin{array}{l}1.80 \\
1.50\end{array}$ & $\begin{array}{l}1.10 \\
1.14\end{array}$ & $\begin{array}{l}.869 \\
.875\end{array}$ & $\begin{array}{l}(.895) \\
(.895)\end{array}$ & $\begin{array}{l}.027 \\
.023\end{array}$ & $\begin{array}{l}.000291 \\
.000290\end{array}$ & Clear, cloudless, humid. \\
\hline $\begin{array}{l}\text { Oct. 23: } \\
9.30 \mathrm{a} . \mathrm{m} \\
10 \mathrm{a} . \mathrm{m} . . . . \\
10.40 \mathrm{a} . \mathrm{m}\end{array}$ & $\begin{array}{l}2.00 \\
1.95 \\
1.83\end{array}$ & $\begin{array}{l}1.19 \\
1.29 \\
1.31\end{array}$ & $\begin{array}{l}.875 \\
.863 \\
.864\end{array}$ & $\begin{array}{l}.893 \\
(.893) \\
(.803)\end{array}$ & $\begin{array}{l}.021 \\
.034 \\
.033\end{array}$ & $\begin{array}{l}.000226 \\
.000407 \\
.000420\end{array}$ & $\begin{array}{l}\text { Sky clear after rain. "City bazo" } \\
\text { in southeast at } 9 \text { to } 10 \mathrm{a} \text {. m. }\end{array}$ \\
\hline $\begin{array}{l}\text { Nov. } 30 \text { : } \\
10.15 \text { a. m..... } \\
10.45 \text { a. m..... }\end{array}$ & $\begin{array}{l}2.38 \\
2.27\end{array}$ & $\begin{array}{r}1.22 \\
1.23\end{array}$ & $\begin{array}{l}.879 \\
.873\end{array}$ & $\begin{array}{l}(.900) \\
.903\end{array}$ & $\begin{array}{l}.023 \\
.033\end{array}$ & $\begin{array}{l}.000219 \\
.000329\end{array}$ & Sky "good." Temperature $16^{\circ} \mathrm{F}$. \\
\hline $\begin{array}{l}\text { Dec. } 3: \\
10.20 \text { a. m..... } \\
10.55 \text { a. } \mathrm{m} \\
\text { Dec. } 20:\end{array}$ & $\begin{array}{l}2.40 \\
2.34\end{array}$ & $\begin{array}{l}.96 \\
.97\end{array}$ & $\begin{array}{l}.888 \\
.885\end{array}$ & $(.901)$ & $\begin{array}{l}.015 \\
.018\end{array}$ & $\begin{array}{l}.000111 \\
.000137\end{array}$ & $\begin{array}{l}\text { Sky "poor," Temperature } 27^{\circ} \mathrm{F} \text {. } \\
\text { windy, sleet on trees. }\end{array}$ \\
\hline $\begin{array}{l}9.50 \mathrm{a} . \mathrm{m} \\
10.25 \mathrm{a} . \mathrm{m}-. . . \\
10.55 \mathrm{a} . \mathrm{m} \\
\end{array}$ & $\begin{array}{l}2.97 \\
2.49 \\
2.37\end{array}$ & $\begin{array}{l}1.15 \\
1.24 \\
1.27\end{array}$ & $\begin{array}{l}.882 \\
.883 \\
.881\end{array}$ & $\begin{array}{l}(.898) \\
.898 \\
(.898)\end{array}$ & $\begin{array}{l}.018 \\
.017 \\
.019\end{array}$ & $\begin{array}{l}.000120 \\
.000155 \\
.000188\end{array}$ & $\left\{\begin{array}{l}\text { Sky clear. Temperature } 25^{\circ} \mathrm{F} \text {. at } \\
10 \text { a. m. Ground covered with } \\
\text { snow. Sky radiation } 6.3 \text { per cent } \\
\text { at } 10.45 \mathrm{a} . \mathrm{m} \text {. }\end{array}\right.$ \\
\hline $\begin{array}{l}\text { Dec. } 21: \\
9.45 \mathrm{a} . \mathrm{m} \\
10.20 \mathrm{a} \cdot \mathrm{m}-\mathrm{m}_{-} \\
10.40 \mathrm{a} \cdot \mathrm{m}-. . \\
1930\end{array}$ & $\begin{array}{l}3.00 \\
2.48 \\
2.40\end{array}$ & $\begin{array}{l}1.17 \\
1.22 \\
1.25\end{array}$ & $\begin{array}{l}.890 \\
.883 \\
.883\end{array}$ & $\begin{array}{l}(.898) \\
.898 \\
(.898)\end{array}$ & $\begin{array}{l}.009 \\
.017 \\
.017\end{array}$ & $\begin{array}{l}.000060 \\
.000155 \\
.000164\end{array}$ & $\left\{\begin{array}{l}\text { Sky cloudless, snow on ground. } \\
\text { Temperature } 25^{\circ} \mathrm{F} \text {. at } 10 \mathrm{8} \text {. } \mathrm{m} \text {. } \\
\text { Sky radiation } 6.5 \text { per cent at } 11 \mathrm{~s} . \mathrm{m} \text {. }\end{array}\right.$ \\
\hline $\begin{array}{l}\operatorname{Jan} .4: \\
10.20 \text { a. } \mathrm{m} \\
10.50 \text { a. } \mathrm{m} \\
\operatorname{Jan} . . . \\
\text { : }\end{array}$ & $\begin{array}{l}2.49 \\
2.44\end{array}$ & $\begin{array}{l}\text { 1. } 30 \\
1.36\end{array}$ & $\begin{array}{l}.886 \\
.881\end{array}$ & . 897 & .012 & $\begin{array}{l}.000115 \\
.000174\end{array}$ & $\left\{\begin{array}{l}\text { Sky cloudless, light wind. Tem. } \\
\text { perature } 34^{\circ} \mathrm{F} \text { at } 10.30 \mathrm{~s} . \mathrm{m} \text {. }\end{array}\right.$ \\
\hline $\begin{array}{l}\text { Jan. } 6: \\
9.40 \mathrm{a} . \mathrm{m} \\
10 \mathrm{a} . \mathrm{m} \\
10.30 \mathrm{a} . \mathrm{m} \\
10.55 \mathrm{a} . \mathrm{m} \\
11.15 \mathrm{a} . \mathrm{m} \\
\text { Feb. } 10 \text { : }\end{array}$ & $\begin{array}{l}3.06 \\
2.80 \\
2.47 \\
2.40 \\
2.34\end{array}$ & $\begin{array}{l}1.12 \\
1.16 \\
1.18 \\
1.20 \\
1.21\end{array}$ & $\begin{array}{l}.887 \\
.892 \\
.878 \\
.873 \\
.864\end{array}$ & $\begin{array}{l}(.898) \\
.898 \\
(.896) \\
.894 \\
(.894)\end{array}$ & $\begin{array}{l}.012 \\
.007 \\
.020 \\
.024 \\
.033\end{array}$ & $\begin{array}{l}.000074 \\
.000052 \\
.000178 \\
.000222 \\
.000314\end{array}$ & $\begin{array}{l}\text { Frosty morning. Sky cloudless in } \\
\text { a. m. Temperature } 40^{\circ} \mathrm{F} \text {. at } \\
10.30 \text { a. m. Sky radiation } 7.2 \text { per } \\
\text { cent at } 10.45 \text { a. m. }\end{array}$ \\
\hline $\begin{array}{l}\text { Feb. } 10: \\
10.15 \mathrm{a} . \mathrm{m} \\
10.30 \mathrm{a} \cdot \mathrm{m} \\
10.45 \mathrm{a} \cdot \mathrm{m} \\
11.30 \mathrm{a} \cdot \mathrm{m} \\
\text { Mar. } 13:\end{array}$ & $\begin{array}{l}2.05 \\
1.99 \\
1.94 \\
1.75\end{array}$ & $\begin{array}{l}1.346 \\
1.355 \\
1.358 \\
1.366\end{array}$ & $\begin{array}{l}.871 \\
.865 \\
.863 \\
.865\end{array}$ & $\begin{array}{l}(.895) \\
.895 \\
(.895) \\
(.895)\end{array}$ & $\begin{array}{l}.027 \\
.033 \\
.036 \\
.034\end{array}$ & $\begin{array}{l}.000322 \\
.000404 \\
.000454 \\
.000468\end{array}$ & $\begin{array}{l}\text { Morning sky cloudless. Falnt cir- } \\
\text { rus at } 11.15 \text { a. m. Sky radiation } 7.8 \\
\text { per cent at } 11 \text { a. m. }\end{array}$ \\
\hline $\begin{array}{l}\text { Mar. 13: } \\
9.05 \mathrm{a} . \mathrm{m} \\
9.25 \mathrm{a} . \mathrm{m} \\
9.55 \mathrm{a} . \mathrm{m} \\
10.45 \mathrm{a} . \mathrm{m} \\
11.50 \mathrm{a} . \mathrm{m} \\
\end{array}$ & $\begin{array}{l}2.16 \\
1.87 \\
1.64 \\
1.48 \\
1.36\end{array}$ & $\begin{array}{l}.978 \\
1.002 \\
1.043 \\
1.144 \\
1.151\end{array}$ & $\begin{array}{l}.887 \\
.873 \\
.873 \\
.869 \\
.869\end{array}$ & $\begin{array}{l}.899 \\
(.896) \\
(.896) \\
.893 \\
(.893)\end{array}$ & $\begin{array}{l}.013 \\
.026 \\
.026 \\
.027 \\
.027\end{array}$ & $\begin{array}{l}.000109 \\
.000249 \\
.000281 \\
.000314 \\
.000367\end{array}$ & $\begin{array}{l}\text { Sky cloudless, moro hazy at } 11.50 . \\
\text { Very hazy in p. m. Sky radiation } \\
10.2 \text { per cent at } 11 \mathrm{a} . \mathrm{m} \text {. }\end{array}$ \\
\hline $\begin{array}{l}\text { Mar. } 15: \\
9.25 \mathrm{a} . \mathrm{m} \\
10.05 \mathrm{a} . \mathrm{m} \\
10.25 \mathrm{a} . \\
11.25 \mathrm{a} . \mathrm{m} \\
11.55 \mathrm{a} . \mathrm{m} \\
\end{array}$ & $\begin{array}{l}1.84 \\
1.59 \\
1.51 \\
1.36 \\
1.33\end{array}$ & $\begin{array}{l}1.054 \\
1.131 \\
1.153 \\
1.198 \\
1.207\end{array}$ & $\begin{array}{l}.871 \\
.871 \\
.861 \\
.861 \\
.860\end{array}$ & $\begin{array}{l}(.895) \\
(.895) \\
.894 \\
(.894) \\
(.894)\end{array}$ & $\begin{array}{l}.027 \\
.027 \\
.037 \\
.037 \\
.038\end{array}$ & $\begin{array}{l}.000274 \\
.000324 \\
.000471 \\
.000523 \\
.000545\end{array}$ & $\begin{array}{l}\text { Sky cloudless in a. } \mathrm{m} \text {. } \\
\text { Sky radiation } 10.8 \text { per cent at } 11.15 \\
\text { a.m. }\end{array}$ \\
\hline
\end{tabular}


TABLE 2.-Ultra-violet solar radiation intensity, $u . v . Q$, of wave lengths less than $313 \mathrm{m \mu}$, also the total sclar radiation intensity, $Q$, in $\mathrm{g} . \mathrm{cal} . / \mathrm{min} ., / \mathrm{cm}^{2}$ for different air masses, $m$, at Washington, D. C. (altitude 350 feet)-Continued

\begin{tabular}{|c|c|c|c|c|c|c|c|}
\hline \multirow{2}{*}{ Date } & \multirow{2}{*}{ Air } & \multirow{2}{*}{$Q$} & \multicolumn{2}{|c|}{$\begin{array}{l}\text { Screen trans- } \\
\text { mission }\end{array}$} & \multirow{2}{*}{$\begin{array}{l}1.000 \\
\text { minus } \\
\text { column } \\
4 / \text { col- } \\
\text { umn } 5\end{array}$} & \multirow{2}{*}{ u. จ. Q } & \multirow{2}{*}{ Remarks } \\
\hline & & & $\frac{a}{I_{0}}$ & $\frac{a+b}{a}$ & & & \\
\hline 1 & $?$ & 3 & 1 & 5 & 6 & 7 & 8 \\
\hline $\begin{array}{l}1929 \\
\text { Mar. } 20: \\
9.05 \mathrm{a} . \mathrm{m} \\
10 \mathrm{a} . \mathrm{m} \\
10.35 \mathrm{a} . \mathrm{m} \\
11.35 \mathrm{a} . \mathrm{m} \\
11.55 \mathrm{a} . \mathrm{m} \\
\text { Apr. } 11 \text { : }\end{array}$ & $\begin{array}{l}m \\
2.00 \\
1.58 \\
1.48 \\
1.37 \\
1.31\end{array}$ & $\begin{array}{l}g \text { cal./ } \\
\mathrm{cm}^{2} / \\
\min _{.} \\
1.104 \\
1.224 \\
1.242 \\
1.274 \\
1.281\end{array}$ & $\begin{array}{l}0.869 \\
.865 \\
.857 \\
.859 \\
.853\end{array}$ & $\begin{array}{l}(0.896) \\
.896 \\
(.895) \\
(.895) \\
.893\end{array}$ & $\begin{array}{l}0.030 \\
.035 \\
.043 \\
.040 \\
.045\end{array}$ & $\begin{array}{c}g \text { cal.l } \\
\text { cm } m^{2} / \mathrm{min} . \\
0.000299 \\
.000459 \\
.000595 \\
.000597 \\
.000690\end{array}$ & $\begin{array}{l}\text { Sky cloudless in } a . \mathrm{m} \text {. Sky radiation } \\
7.6 \text { per cent at } 11.50 \mathrm{a} . \mathrm{m} \text {. }\end{array}$ \\
\hline $\begin{array}{l}10.15 \text { a. m } \\
12.20 \text { p. m } \\
2.45 \text { p. } \mathrm{m} . . .\end{array}$ & $\begin{array}{l}1.31 \\
1.20 \\
1.58\end{array}$ & $\begin{array}{l}.87 \\
.90 \\
.64\end{array}$ & $\begin{array}{l}.881 \\
.869 \\
.881\end{array}$ & $\begin{array}{l}.897 \\
(.897) \\
(.897)\end{array}$ & $\begin{array}{l}.028 \\
.031 \\
.028\end{array}$ & $\begin{array}{l}.000292 \\
.000346 \\
.000192\end{array}$ & $\begin{array}{l}\text { Thick haze with cirri at } 10.40 \mathrm{a} . \mathrm{m} \text {. } \\
\text { to } 12.15 \mathrm{p} . \mathrm{m} \text {. Temperature } 75^{\circ} \mathrm{F} \\
\text { Sky radiation } 17.0 \text { per cent at } 10.30 \\
\text { a. } \mathrm{m} \text {. }\end{array}$ \\
\hline $\begin{array}{l}\text { Apr. } 12: \\
\quad 9.20 \text { a. m...... } \\
10 \mathrm{a} . \mathrm{m} \\
\quad 1.15 \mathrm{p} . \mathrm{m} . \ldots \\
\text { May } 3:\end{array}$ & $\begin{array}{l}1.55 \\
1.35 \\
1.24\end{array}$ & $\begin{array}{l}.52 \\
.74 \\
.78\end{array}$ & $\begin{array}{l}.885 \\
.874 \\
.875\end{array}$ & $\begin{array}{l}.902 \\
(.900) \\
.898\end{array}$ & $\begin{array}{l}.019 \\
.029 \\
.026\end{array}$ & $\begin{array}{l}.000107 \\
.000253 \\
.000246\end{array}$ & $\begin{array}{l}\text { Thick haze; forest fires on } \mathrm{Apr} \text {. } 11 \text { and } \\
12 . \text { Sky radiation } 19.0 \text { per cent at } \\
1.05 \text { p. m. }\end{array}$ \\
\hline $\begin{array}{r}9.05 \mathrm{a} . \mathrm{m} \\
10.05 \mathrm{a} . \mathrm{m} \\
11.05 \mathrm{a} . \mathrm{m} \\
12.05 \mathrm{p} . \mathrm{m} \\
1.40 \mathrm{p} . \mathrm{m} \\
2.20 \mathrm{p} . \mathrm{m} \\
\text { May } 5 \text { : }\end{array}$ & $\begin{array}{l}1.42 \\
1.36 \\
1.24 \\
1.20 \\
1.29 \\
1.36\end{array}$ & $\begin{array}{l}1.29 \\
1.30 \\
1.31 \\
1.32 \\
1.30 \\
1.29\end{array}$ & $\begin{array}{l}.850 \\
.845 \\
.841 \\
.843 \\
.849 \\
.855\end{array}$ & $\begin{array}{l}.887 \\
(.887) \\
.889 \\
(.889) \\
(.890) \\
.890\end{array}$ & $\begin{array}{l}.042 \\
.047 \\
.054 \\
.052 \\
.045 \\
.039\end{array}$ & $\begin{array}{l}.000622 \\
.000721 \\
.000858 \\
.000850 \\
.000716 \\
.000594\end{array}$ & $\begin{array}{l}\text { Sky cloudless; clearer in p. m. Sky } \\
\text { radiation } 8.8 \text { per cent at } 9.35 \text { a. m.; } \\
8.2 \text { per cent at } 10.55 \text { a. } \mathrm{m} \text {. }\end{array}$ \\
\hline 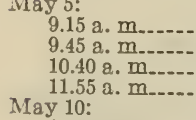 & $\begin{array}{l}\text { 1. } 40 \\
1.37 \\
1.26 \\
1.20\end{array}$ & $\begin{array}{l}1.05 \\
1.13 \\
\text { l. } 18 \\
1.20\end{array}$ & $\begin{array}{l}.875 \\
.876 \\
.863 \\
.859\end{array}$ & $\begin{array}{l}.893 \\
(.893) \\
(.893) \\
(.893)\end{array}$ & $\begin{array}{l}.020 \\
.019 \\
.034 \\
.038\end{array}$ & $\begin{array}{l}.000243 \\
.000252 \\
.000487 \\
.000565\end{array}$ & $\begin{array}{l}\text { Sky thick, hazy; forest fires. Sky } \\
\text { radiation } 11.6 \text { per cent at } 10.30 \text { a. m. } \\
\text { More hazy than May } 3 .\end{array}$ \\
\hline $\begin{array}{l}9.15 \text { a. m... } \\
10 \text { a. m. } \\
10.55 \mathrm{a} . \mathrm{m} \\
12.02 \mathrm{p} . \mathrm{m} \\
12.20 \mathrm{p} . \mathrm{m} \\
1.50 \mathrm{p} . \mathrm{m} \\
2.25 \mathrm{p} . \mathrm{m} \\
\text { June } 2 \text { : }\end{array}$ & $\begin{array}{l}1.38 \\
1.34 \\
1.23 \\
1.19 \\
1.20 \\
1.33 \\
1.36\end{array}$ & $\begin{array}{l}1.12 \\
1.20 \\
1.23 \\
1.27 \\
1.26 \\
1.26 \\
1.25\end{array}$ & $\begin{array}{l}.860 \\
.860 \\
.848 \\
.846 \\
.847 \\
.850 \\
.855\end{array}$ & $\begin{array}{l}(.892) \\
.892 \\
(.892) \\
.886 \\
(.886) \\
(.883) \\
.883\end{array}$ & $\begin{array}{l}.036 \\
.036 \\
.049 \\
.045 \\
.044 \\
.037 \\
.032\end{array}$ & $\begin{array}{l}.000469 \\
.000510 \\
.000736 \\
.000708 \\
.000687 \\
.000554 \\
.000472\end{array}$ & $\begin{array}{l}\text { Sky cloudless all day. Light SE. } \\
\text { wind. Sky radiation } 9.2 \text { per cent } \\
\text { at } 10.35 \text { a. m.; sky radiation to } \\
\text { WSW. } 1.9 \text { per cent; zenith, } 3.3 \text { per } \\
\text { cent at } 10.50 \text { a. m., to SE. } 2.6 \text { per } \\
\text { cent at } 3.15 \text { p. m. }\end{array}$ \\
\hline $\begin{array}{l}9.30 \mathrm{a} \cdot \mathrm{m} \\
9.55 \mathrm{a} \cdot \mathrm{m} \\
12.10 \mathrm{p} \cdot \mathrm{m} . \\
2.05 \mathrm{p} \cdot \mathrm{m}\end{array}$ & $\begin{array}{l}1.25 \\
1.18 \\
1.05 \\
1.13\end{array}$ & $\begin{array}{l}\text { 1. } 22 \\
1.32 \\
1.30 \\
1.28\end{array}$ & $\begin{array}{l}.858 \\
.853 \\
.845 \\
.852\end{array}$ & $\begin{array}{l}(.890) \\
.890 \\
(.890) \\
(.890)\end{array}$ & $\begin{array}{l}.036 \\
.042 \\
.051 \\
.043\end{array}$ & $\begin{array}{l}.000533 \\
.000691 \\
.000871 \\
.000700\end{array}$ & $\left\{\begin{array}{l}\text { Sky cloudless in a. m. Sky radiation } \\
7.5 \text { per cent at } 10.30 \text { and } 7.8 \text { per cent } \\
\text { at } 11.30 \mathrm{a} . \mathrm{m} \text {. Sky SW. } 2.8 \text { per cent } \\
\left.\text { (altitude } 40^{\circ}\right) \text {. Ratio } a \div I_{0} \text { for } \mathrm{SW} \text {. } \\
\text { sky }=0.78 \text { to } 0.81 \text { showing greater } \\
\text { amount of ultra-Violet. Thick cir- } \\
\text { ri at } 2.10 \mathrm{p} . \mathrm{m} \text {. }\end{array}\right.$ \\
\hline $\begin{array}{l}9.55 \mathrm{a} . \mathrm{m} \\
10.15 \mathrm{a} . \mathrm{m} \\
11.10 \text { a. } \mathrm{m} \\
12.01 \mathrm{p} . \mathrm{m}_{-} \\
1.30 \mathrm{p} . \mathrm{m} \\
\text { July } 7:\end{array}$ & $\begin{array}{l}\text { 1. } 24 \\
\text { 1. } 11 \\
1.07 \\
1.05 \\
\text { 1. } 10\end{array}$ & $\begin{array}{l}1.27 \\
1.33 \\
1.35 \\
1.37 \\
1.32\end{array}$ & $\begin{array}{l}.855 \\
.850 \\
.847 \\
.845 \\
.849\end{array}$ & $\begin{array}{l}(.887) \\
.887 \\
(.887) \\
.887 \\
(.887)\end{array}$ & $\begin{array}{l}.036 \\
.042 \\
.045 \\
.047 \\
.043\end{array}$ & $\begin{array}{l}.000555 \\
.000720 \\
.000794 \\
.000835 \\
.000731\end{array}$ & $\begin{array}{l}\text { Sky cloudless. Temperature } 86^{\circ} \mathrm{F} \text {. } \\
\text { at noon. Sky radiation } 8.1 \text { per cent } \\
\text { at } 11.45 \mathrm{a} . \mathrm{m} \text {. }\end{array}$ \\
\hline $\begin{array}{l}9.45 \text { a. m-.. } \\
10.30 \text { a. m-. } \\
\text { July } 19:\end{array}$ & $\begin{array}{l}\text { 1. } 40 \\
1.21\end{array}$ & $\begin{array}{l}\text { 1. } 19 \\
1.23\end{array}$ & .853 & $\begin{array}{l}.884 \\
(.884)\end{array}$ & .035 & .000481 & Sky cloudless after heavy rain. \\
\hline $\begin{array}{l}9.10 \text { a. } \mathrm{m}_{-. .} \\
10.20 \mathrm{a} \cdot \mathrm{m}_{-. .} \\
10.35 \mathrm{a} \cdot \mathrm{m} \\
\text { July } 21:\end{array}$ & $\begin{array}{l}1.42 \\
1.25 \\
1.22\end{array}$ & $\begin{array}{l}1.05 \\
1.14 \\
1.19\end{array}$ & $\begin{array}{l}.870 \\
.850 \\
.850\end{array}$ & $\begin{array}{l}.893 \\
.890 \\
.887\end{array}$ & $\begin{array}{l}.026 \\
.045 \\
.042\end{array}$ & $\begin{array}{l}.000316 \\
.000623 \\
.000615\end{array}$ & $\left\{\begin{array}{l}\text { Fine cirri SE. of sun. Sky radiation } \\
10.1 \text { per cent at } 10.45 \mathrm{a} . \mathrm{m} \text {. }\end{array}\right.$ \\
\hline $\begin{array}{l}10.35 \text { a. } \mathrm{m}_{--} \\
11.00 \text { \%. } \mathrm{m} \\
11.40 \text { a. } \mathrm{m} \\
\text { July } 25:\end{array}$ & $\begin{array}{l}\text { 1. } 23 \\
1.17 \\
1.10\end{array}$ & $\begin{array}{l}\text { 1. } 09 \\
1.13 \\
1.16\end{array}$ & $\begin{array}{l}.857 \\
.859 \\
.861\end{array}$ & $\begin{array}{l}.892 \\
(.892) \\
(.892)\end{array}$ & $\begin{array}{l}.039 \\
.037 \\
.035\end{array}$ & $\begin{array}{l}.000519 \\
.000525 \\
.000523\end{array}$ & $\begin{array}{l}\text { Sky hazy. Hot, } 100^{\circ} \text { F. Sky radi- } \\
\text { ation } 16.5 \text { per cent at } 10.50 \text { a. m. }\end{array}$ \\
\hline $\begin{array}{l}10.35 \text { a. } \mathrm{m} \\
10.50 \mathrm{a} \cdot \mathrm{m} \\
11.45 \mathrm{a} \cdot \mathrm{m} \\
\text { Aug. } 12:\end{array}$ & $\begin{array}{l}\text { 1. } 24 \\
1.17 \\
1.09\end{array}$ & $\begin{array}{l}\text { 1. } 17 \\
\text { 1. } 21 \\
\text { 1. } 23\end{array}$ & $\begin{array}{l}.850 \\
.852 \\
.844\end{array}$ & $\begin{array}{l}(.890) \\
.890 \\
(.890)\end{array}$ & $\begin{array}{l}.045 \\
.043 \\
.052\end{array}$ & $\begin{array}{l}.000639 \\
.000653 \\
.000825\end{array}$ & $\begin{array}{l}\text { Sky clear; hot, } 98^{\circ} \mathrm{F} \text {. at noon. Sky } \\
\text { radiation } 8.3 \text { per cent at } 11 \mathrm{a.} \mathrm{m.}\end{array}$ \\
\hline 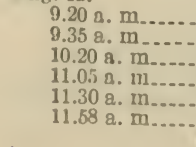 & $\begin{array}{l}1.40 \\
1.38 \\
1.21 \\
1.14 \\
1.11 \\
1.10\end{array}$ & $\begin{array}{l}\text { 1. } 22 \\
\text { 1. } 23 \\
\text { 1. } 27 \\
\text { 1. } 27 \\
\text { 1. } 29 \\
1.28\end{array}$ & $\begin{array}{l}.857 \\
.852 \\
.846 \\
.842 \\
.854 \\
.851\end{array}$ & $\begin{array}{l}.886 \\
(.886) \\
(.885) \\
.890 \\
(.890) \\
(.830)\end{array}$ & $\begin{array}{l}.033 \\
.033 \\
.045 \\
.054 \\
.041 \\
.014\end{array}$ & $\begin{array}{l}.000465 \\
.000544 \\
.000708 \\
.000866 \\
.000676 \\
.000726\end{array}$ & $\begin{array}{l}\text { Forenoon sky cloudless. Thick hazo } \\
\text { in west and south horizon at noon. } \\
\text { Cool, } 72^{\circ} \mathrm{F} \text {. at noon. Sky radia- } \\
\text { tion } 7 \text { per cent at } 11 \mathrm{a} \text {. } \mathrm{m} \text {. }\end{array}$ \\
\hline
\end{tabular}


The difficulty arises in expressing these relative values in absolute units of energy. No screen or filter is available that has a sharply defined cut-off at a definite wave length. The effective cut-off of one screen that is opaque to wave lengths less than $310 \mathrm{~m} \mu$ (for example, the barium flint screens used in this work, which has the sharpest cut-off yet found) ranges from about $338 \mathrm{~m} \mu$ for air mass, $m=1$ to $341 \mathrm{~m} \mu$ for $m=3$. This, of course, was known at the beginning of the investigation and recently it has been discussed in print (3).

Owing to the fact that the effective cut-off does not coincide with the long wave-length limit (about $313 \mathrm{~m} \mu$ ) of the biologically active ultra-violet radiation, the observed values (in per cent of the total in column 6 of Tables 1 and 2) obtained by this exclusion method of radiometry, are larger than the true values. The difference between these two values could be determined from a knowledge of the solar spectral energy curve using only one filter. Unfortunately, the ultraviolet spectral energy curve, even for average atmospheric conditions is not well known, and in the spectral region of wave lengths less than $313 \mathrm{~m} \mu$ it is in greater doubt than for wave lengths 313 to about 365 $\mathrm{m} \mu$ (the latter value being the long wave length limit concerned in the barium flint screen measurement).

In order to obviate these difficulties to some extent, measurements were made with one $(a)$ and with two, $(a+b)$ filters of barium flint glass interposed; the latter to determine the percentage of radiation of wave lengths longer than $313 \mathrm{~m} \mu$ transmitted by the first glass.

As will be noted presently. for the sea-level station, there is a reasonably close agreement between the observed and the computed transmissions. (See fig. 3.) For the higher elevations there is considerable divergence between the calculated and the observed transmissions. (See fig. 4.)

Another difficulty in evaluating the ultra-violet component of solar radiation at 295 to $313 \mathrm{~m} \mu$, based upon calculations using the spectral energy curve of the sun, is owing to the fact that, in all such calculations, a smooth energy curve is used, whereas the observed curve is deeply indented by absorption bands. Moreover, the spectral energy curve in the ultra-violet, especially of wave lengths less than $313 \mathrm{m \mu}$ is greatly affected by slight changes in atmospheric pollution. This is well illustrated in the transmission measurements of solar radiation through one filter of barium flint glass, which values vary greatly from day to day (as compared with the transmissions of the second filter) for solar radiation of wave lengths longer than $313 \mathrm{~m} \mu$ that has passed through the first filter, which values vary but little with change in air mass and atmospheric pollution. Hence, as already indicated, the second filter is useful not only in measuring the ultra-violet energy between $313 \mathrm{~m} \mu$ and about $365 \mathrm{~m} \mu$; but, since the ultra-violet cut-off is not sharply defined, it is useful also in obtaining a factor for eliminating the amount of ultra-violet of wave lengths longer than 313 $\mathrm{m} \mu$ included in the measurement of the ultra-violet of wave lengths less than $313 \mathrm{~m} \mu$, obtained with the single filter. This fact, as already stated, was recognized from the start, but the complete correction was not included in the previous paper (2) where only the upper limiting value of the ultra-violet (in per cent of the total) was derived.

The solar spectral energy curves used in the present calculations of the Washington data were taken from the Smithsonian Physical Tables, supplemented in the extreme ultra-violet by the data pub- 
lished by O'Brien, by Fabry, and Buisson (4) and by Greider and Downs (5).

The manner of obtaining the ultra-violet radiation of wave lengths less than $313 \mathrm{~m} \mu$, by means of this 2-screen method, is illustrated in Figure 2 in which curve $A$ is the observed solar spectral energy curve $(m=1)$; curve $B$ gives the spectral energy distribution, $I_{0}$, after passing through the Corning glass filter, G986A; curve $C$ shows the spectral energy distribution after transmission through one plate of barium flint glass $(\mathrm{G} 669, a)$ and curve $D$ shows the spectral energy distribution after passing through the two filters $(\mathrm{G} 669, a+b)$.

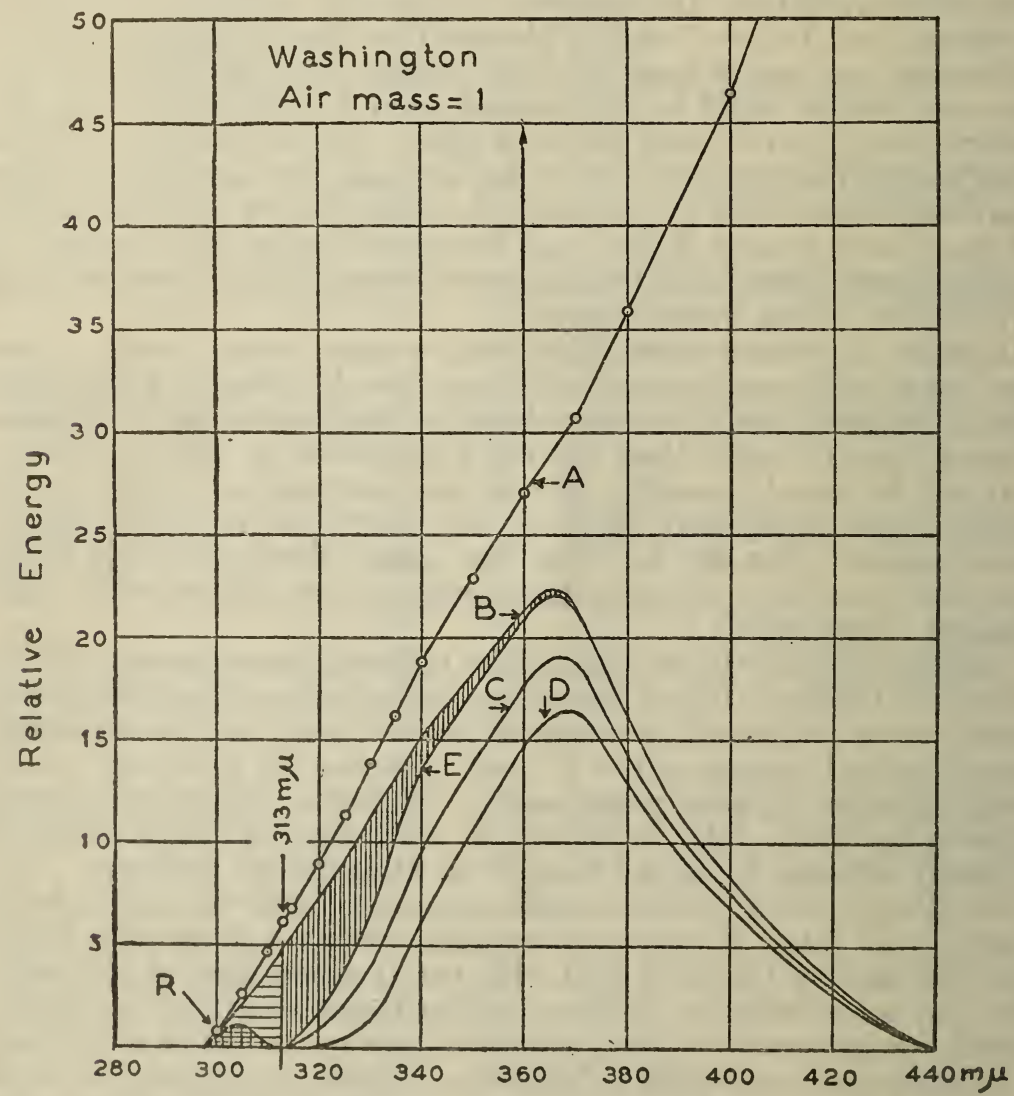

FIGURE 2.-Graphical method used in reducing the data

The whole shaded area between curves $B$ and $E$ represents the ultra-violet spectral energy (column 6 in Tables 1 and 2 ) excluded by this 2 -screen method of making the measurement, whereas, the horizontally shaded area, of wave lengths less than $313 \mathrm{~m} \mu$, represents the ultra-violet energy that it is desired to evaluate. Incidentally, it may be noted that the effective wave length cut-off, using two screens, occurs at about $329 \mathrm{~m} \mu$ for air mass $m=1$ to 330.5 $\mathrm{m} \mu$ for $m=3$. It is the sharpest cut-off of all the screens yet tested.

For the sea-level station (Washington, altitude $=350$ feet) the calculated per cent transmissions for the single barium flint screen, 
indicated by the symbol $a / I_{\mathrm{o}}$, and for the two screens, symbol $(a+b) / a$ (fig. 3) are in as close agreement as can bo expected. The ratios of observed and calculated values $\left[a / I_{0}:(a+b) / a\right]$ are in much closer agreement. In view of this close agreement between. the calculated and the observed data, it is assumed permissible to calculate suitable factors, $F$, from the average spectral energy curves, in order to reduce the observed measurements (given in column 6 of Tables 1 and 2) represented by the whole shaded area between cirves $B$ and $E$, to the energy represented by the horizontally shaded area comprised of wave lengths less than $313 m \mu$ (shown in fig. 2) which energy, as just mentioned, it is desired to evaluate.

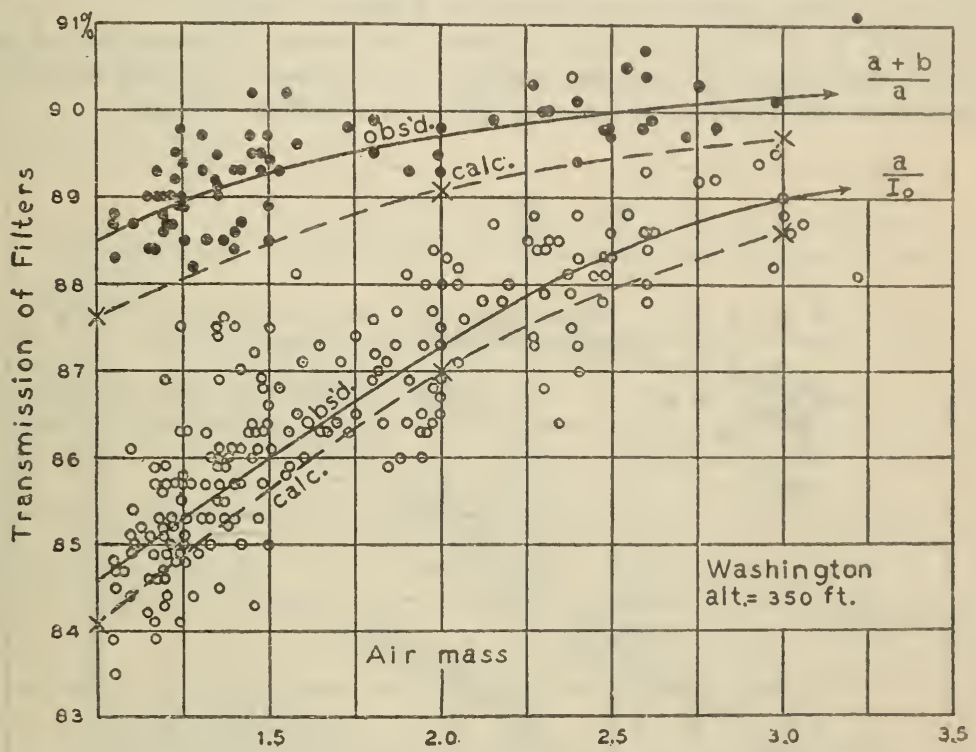

Figure 3.-Observed and calculated transmissions of one and of two standard barium flint glass filters, for different air masses, at Washington, September, 1929, to January, 1931

These factors, $F$, are obtained graphically from the averagefsolar spectral energy curves, for air masses $m=1,2$, and 3 , in which the area, $I_{0}$, represents the radiation measured after transmission through the combined filter consisting of the water cell and the purple glass screen, G986A. Similar curves were obtained to represent the spectral transmissions $a / I_{0}$ and $(a+b) / a$, after inscrtion of the barium flint screens $a$, and $a+b$. The ultra-violet ends of these curves representing $I_{0}, a / I_{0}$ and $(a+b) / a$ are shown in curves $B, C$, and $D$ in Figure 2.

Since these screens have no selective absorption in the infra-red (which is terminated at $1.4 \mu$ by the water cell) the determination of the reduction factors, $F$, involves an accurate integration of only the ultra-violet areas of the curves $B, C, D$, and $E$, and of the shaded area of wave lengths less than $313 \mathrm{~m} \mu$, depicted in Figure 2.

These correction factors, $F$, for different air masses are shown graphically in Figure 5, from which curve is taken the value for the particular air mass through which the measurements were made. 
The observed amount of ultra-violet shut out by the barium flint glass screens (given in column 6 of Tables 1 and 2 ; these values are 1.000 - columns $4 \div 5$ ) is multiplied by the corresponding factor, $F$ (fig. 5), corresponding with the air mass, $m$, given in column 2 of Tables 1 and 2 , in order to obtain the amount of ultra-violet comprised in the band of wave lengths less than $313 \mathrm{~m} \mu$.

Since all these values are in terms of the total incident solar radiation intensity, $Q$ (g cal. $/ \mathrm{cm}^{2} / \mathrm{min}$.) and of the transmission factor $K$ $(=0.0837)$ of the combined water cell and glass screen, G986A, the component ultra-violet solar radiation, $u$. $v$. $Q$., of wave lengths less than $313 \mathrm{~m} \mu$ is the product of $K . Q . F$ and of the values of the ultraviolet part of $Q$, given in column 6 of Tables 1 and 2 .

Column 7 of Tables 1 and 2 gives the total ultra-violet of wave lengths less than $313 \mathrm{~m} \mu$, in sunlight, as deduced by this method of observation and reduction of the data to absolute values.

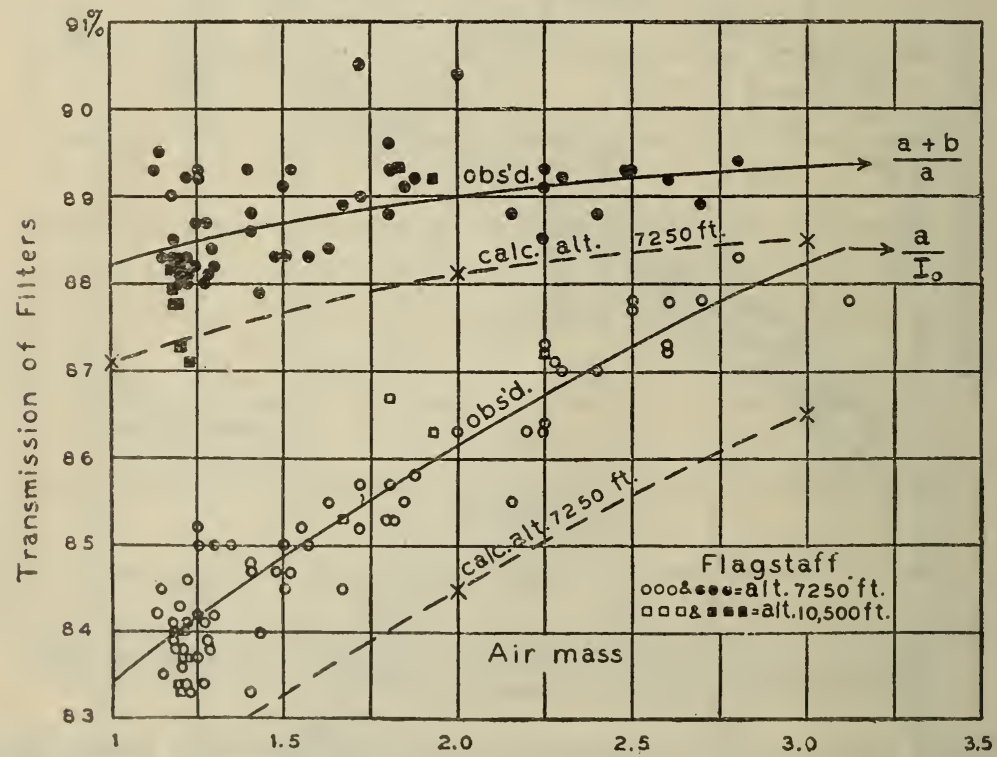

FIgURE 4.-Observed and calculated transmissions of barium fint glass filters for different air masses, at Flagstaff, Ariz., August to September, 1929

In view of the great fluctuations in the ultra-violet component from day to day (see figs. 3 and 4 ), the logical procedure would be to use correction factors, $F$, representing these extremes. However, since the corresponding spectral energy curves are not known it seems sufficient to use the average solar spectral energy curve.

$\Lambda$ s shown in Figure 4 the values of $a / I_{0}$ and of $(a+b) / a$, calculated for high elevations, are much lower than the observed values. The ratios of these two measurements are in still greater disagreement with the observed values, indicating that the solar spectral energy curves, used in the calculations, are richer in short wave length ultraviolet radiation than obtains during actual observations. This is entirely different from the conditions at sea level where the observed and the calculated ratios $\left(a / I_{0}:(a+b) / a\right)$ are in fairiy good agreement. 
The factors, $F$, for reducing the Flagstaff observations to value for the spectral band of wave lengths less than $313 \operatorname{in} \mu$ were, therefore, obtained in the following manner. Instead of air masses the equivalent pressures, in millimeters of mereury (see fig. 5), were plotted against the correction factors, $F$, calculated for Washington (pressure $=760 \mathrm{~mm}$ ). From this curve were read tho correction factors for the two Flagstaff stations (pressures 580 and $500 \mathrm{~mm}$, respectively) for air masses, $m=1,2$, and 3 .

In view of the fact that the total solar radiation intensity, $Q$, obtained by meteorologists, is in $\mathrm{g} \mathrm{cal} . / \mathrm{cm}^{2} / \mathrm{min}$., the present data are given in this unit. The custom among physicists and physiologists is to evaluate thermal radiation flux in radiant power, usually in milliwatts or microwatts $(\mu \mathrm{w})$ per $\mathrm{cm}^{2}$. The data in Tables 1 and 2 may be reduced to watts per $\mathrm{cm}^{2}$ by applying the approximate factor $0.07(=4.18 \div 60)$. They are given in gram-calories and in microwatts in the illustrations.

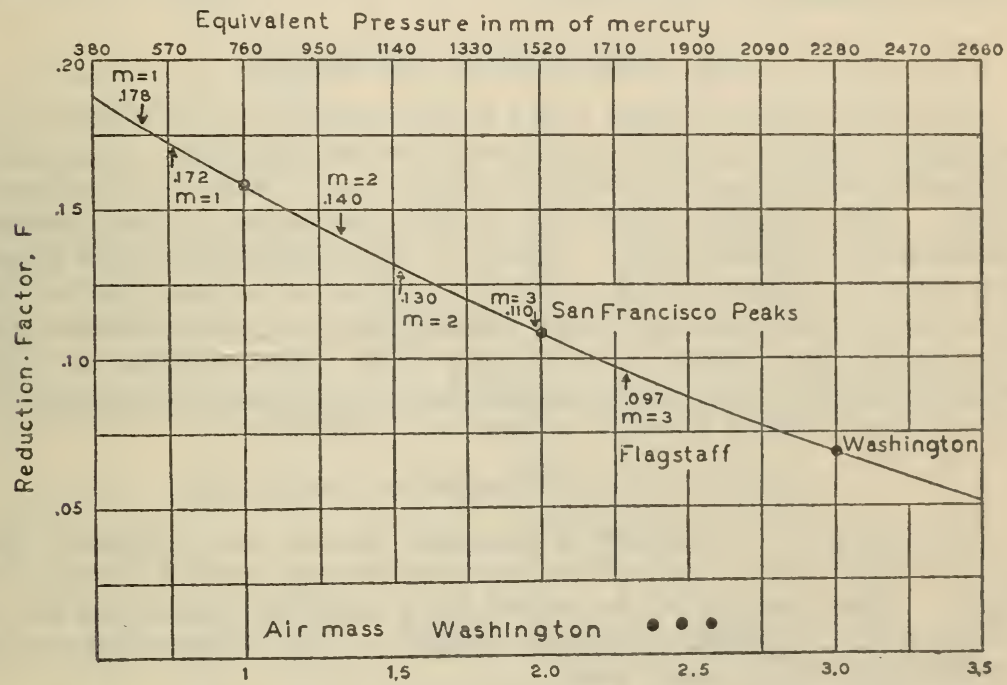

Figure 5.-Reduction factors used in determining the ultra-violet radiation of wave lengths less than $313 \mathrm{m \mu}$

The data given in column 7 of Tables 1 and 2, represent all the ultra-violet solar energy available in the spectral band of wave lengths less than $313 \mathrm{~m} \mu$, generally accepted by biologists as having therapeutic properties, especially in preventing and healing rickets. But the biological effectiveness for equal energy, is not the same for all wave lengths, being a maximum at about $297 \mathrm{~m} \mu$, and decreasing to an extremely low value at $313 \mathrm{~m} \mu$ (6). The spectral erythemic response curve, for equal energies, observed by Hausser and Vahle, by Luckiesh, Holladay, and Taylor (7) and by the writers (data unplublished) is a maximum at $297 \mathrm{~m} \mu$ descreasing to an extremely low value at wave length $313 \mathrm{~m} \mu$.

The rubescence curve ( $R$ in fig. 2 ), which is analagous to the luminosity curve of the eye, is the spectral erythemic response curve for an equal energy spectrum multipled by the spectral energy dis-

$55946^{\circ}-31-4$ 
tribution of the source, which, in this case, is the sun (8). The doubly shaded area under the rubescence curve, $R$, in Figure 2 represents the spectral effectiveness of ultra-violet solar radiation in producing an erythema. The ratio of the shaded area under the rubescence curve $R$, relative to the total shaded area of wave lengths less than $313 \mathrm{~m} \mu$, at sea level, is the erythemic efficiency. This ratio does not seem to vary greatly with air mass, the calculated values being 14.5, 14.0, and 12.8 per cent, respectively, for the corresponding air masses, $m=1,2$, and 3. In other words, the biologically effective ultra-violet component, $R$, is about (14 to 13 per cent) one-seventh to one-eighth of the total ultra-violet component, $u . v$. Q., recorded in column 7 of Tables 1 and 2 ; or a range of about 8 microcalories per $\mathrm{cm}^{2}$ per minute, for $m=3$ to about 125 microcalories per $\mathrm{cm}^{2}$ per minute for $m=1.05$. Expressed in terms of radiant power these values are approximately 0.6 and 9 microwatts per $\mathrm{cm}^{2}$, respectively, showing the extremely small amount of ultra-violet energy useful for thereapeutic purposes that is contained in solar radiation.

\section{DISCUSSION OF DATA}

The data given in Tables 1 and 2 when plotted to scale show interesting information, some of which will now be discussed. A complete discussion of the measurements would be too tedious for the general reader. The tabulated data and remarks can be left to the specialists interested in phototherapy. Many of the phenomena have already been noted by others, but not always recorded on a quantitative basis. Since the present data are based upon measurements that were made only during the clearest, cloud-free weather, the averages are necessarily higher than the values that are based upon measurements which are obtained during all sorts of weather conditions.

\section{EFFECT OF ATMOSPHERIC POLLUTION}

By armospheric pollution is generally meant the temporary presence of dust, smoke, etc. The dust may be transported from an arid region. The smoke may be caused by a neighborhood brush fire, by a forest fire, a passing railroad engine, or the exhaust gases from automobiles, "city haze," etc.

Calculations of the sea-level solar spectral energy curve from data obtained at high elevations seems to indicate the presence of a greater amount of ultra-violet than actually observed, just as though there is a local atmospheric pollution not accounted for in the average spectral energy curve. This, of course, is to be expected.

Notable examples of unusual atmospheric pollution by automobiles, which affected the Washington measurements, are October 8 and 23, 1929 , when the transmission of short-wave length ultra-violet solar radiation was doubled in the course of about 15 minutes. (See Table 2.) The low value of the total solar radiation intensity, $Q$, and of the ultra-violet component, $u . v . Q$., observed on October 9, 1929, are equally interesting.

At the Lowell Observatory station on several occasions the measurements were interrupted by smoke from wood that was burned in a sawmill some miles away. The interesting part of the phenomenon was that on one occasion the blue color of the smoke so closely matched the intensely blue color of the sky that, in the bright sunshine, its presence could hardly be distinguished and would have 
escaped notice if its had not affected the thermopile readings.

Table 1, measurements of 9.55 a. m., Sept. 13, 1929.)

(See

The great variation in the ultra-violet component of solar radiution, as a result of variations in sky conditions, are shown in the high altitude observations (fig. 6) and to a greater extent in the ubservations at Washington. (Fig. 7.) At the latter station, on some days, the ultra-violet component for air mass, $m=1.5$, is not much larger than in the morning and late afternoon of the clearest days, air mass, $m=3$.

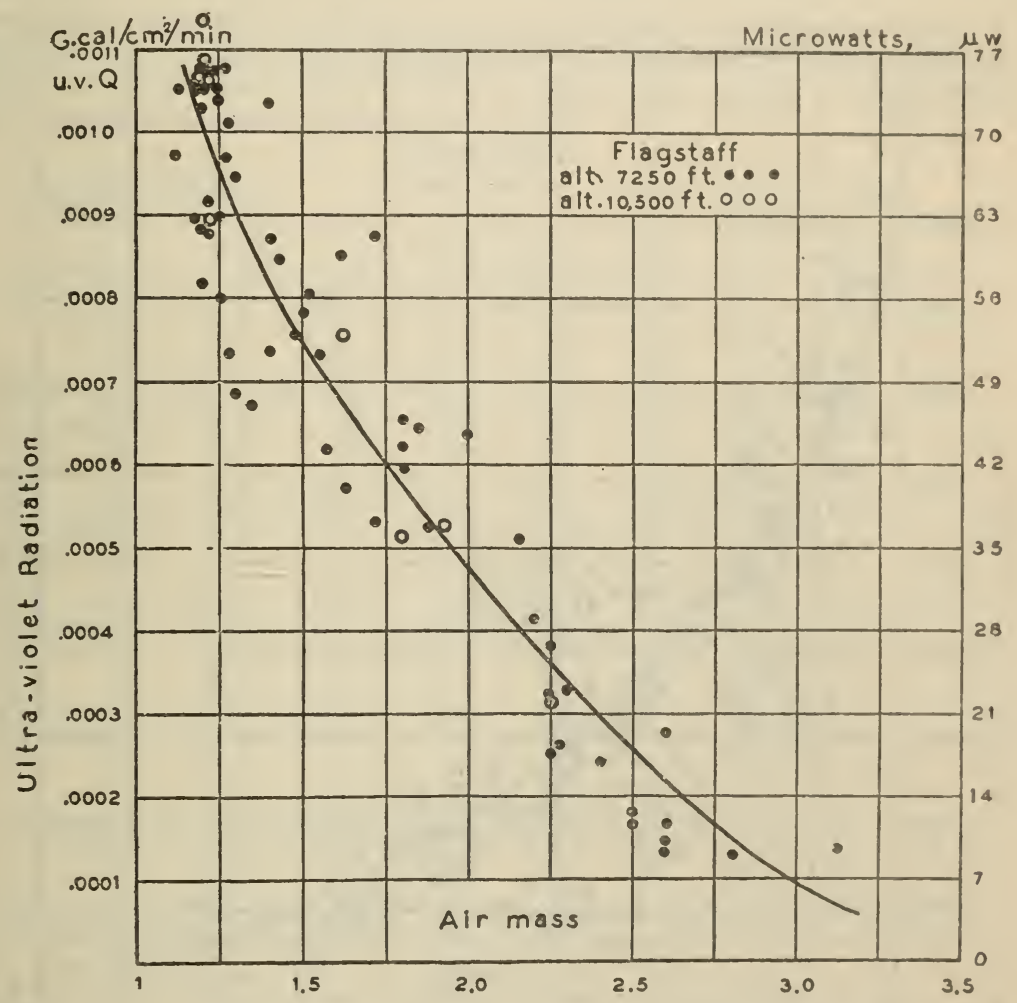

Figdre 6.-Total ultra-violet solar radiation of wave lengths less than 313 $m \mu$, in $\mathrm{g}$ cal. per $\mathrm{cm}^{2}$ per min. (also in microwatts, $\mu w$, pei $\mathrm{cm}^{2}$ ), for different air masses at Flagstaff, August and September, 1929

As is to be expected, for a given air mass, the greater the amount of scattering of the incident rays, the smaller the amount of the directly incident ultra-violet solar radiation, $u . v . Q$. This is illustrated in Figure $8(m=1.25)$ in which is plotted the total ultra-violet component, $u$. v. Q., against the per cent of (scattered) "sky radiation," present in the total incident ultra-violet solar radiation measured.

\section{EFFECT OF ALTITUDE}

The effect of altitude on the short-wave length ultra-violet component of sunlight is well known, though quantitative data are not numerous. 
Two outstanding factors contribute to the amount of ultra-violet received. The first factor is that, with rise in elevation, the spectral absorption is decreased and the intensity of the short wave lengths, $300 \mathrm{~m} \mu$ relative to $320 \mathrm{~m} \mu$, is greatly increased. This is indicated in the lower values of the transmission of the barium flint giass screens, $a / I_{0}$ and $(a+b) / a$, in going from the 7,250 feet elevation to the 10,500 feet elevation.

The second factor is the amount contributed by atmospheric scattering along the path of the incident rays. In this connection Pettit

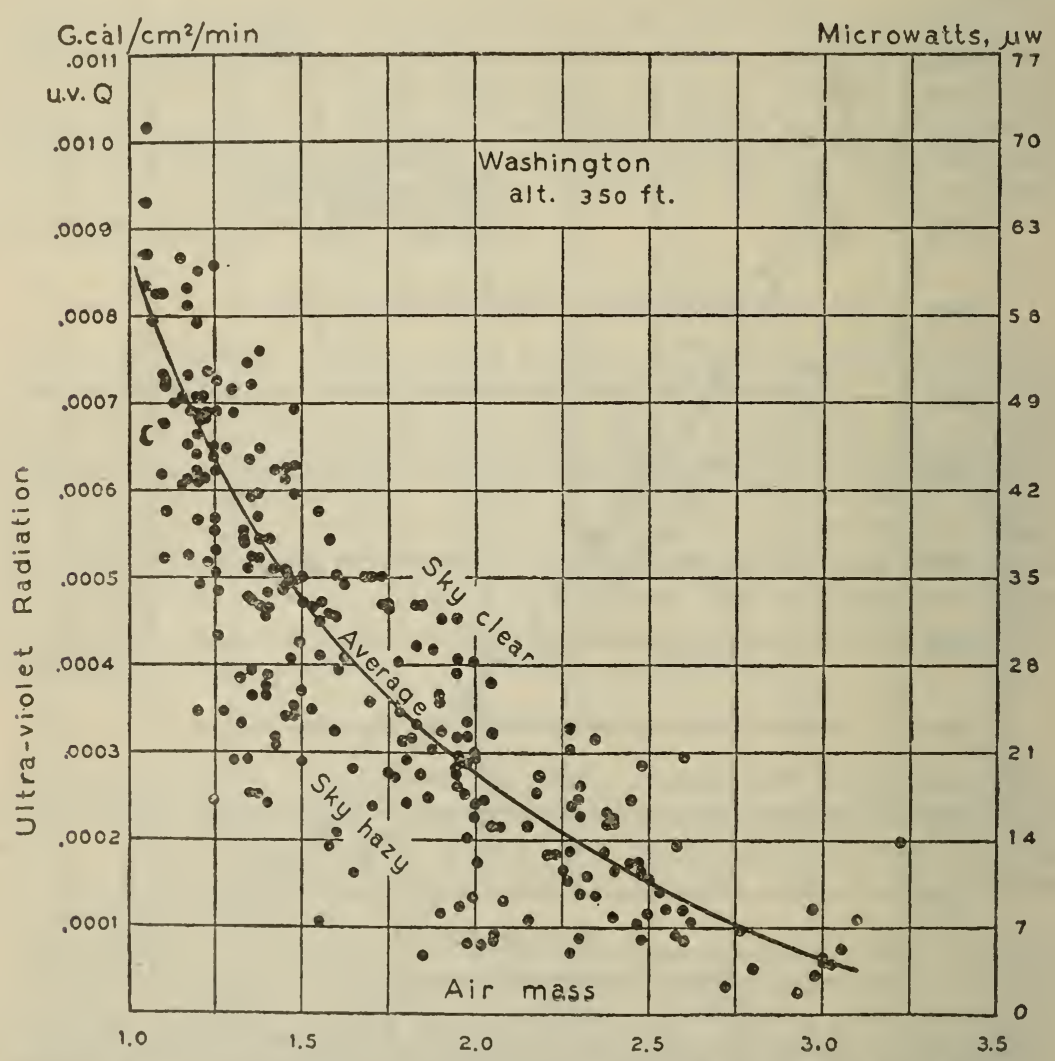

ligure 7.-Total ultra-violet solar radiation, of wave lengths less than $818 \mathrm{m \mu}$, in $\mathrm{g}$ cal. per $\mathrm{cm}^{2}$ per minute (also in microwatts per $\mathrm{cm}^{2}$ ), for different air masses at Washington, September, 1929, to January, 1931

(9) has recently contributed some interesting data, showing that, in going from a sea-level station to a mountain top, the total amount of ultra-violet radiation from the sun increases to some extent, but the ultra-violet scattered by the sky is greatly decreased. His measurements were made at $320 \mathrm{~m} \mu$, at which wave length, as shown by the transmission factors $(a+b) / a$, the effect of scattering, with air mass and change in altitude, is less marked than for the shorter wave lengths (at $300 \mathrm{~m} \mu$ ) involved in the present measurements using the single filter, $a / I_{0}$. 
Using a photo-electric cell covered with a filın of silver, which transmits ultra-violet of wave lengths at $320 \mathrm{~m} \mu$, Pettit (9) found that about 50 per cent of the ultra-violet observed at an elevation of 845 feet was contributed by the sky, while at an elevation of 5,700 feet only about 30 per cent of the total ultra-violet measured was contributed by the sky. On the other hand, the total ultra-violet (sun plus sky) observed at the 5,700-foot elovation was only about 5 per cent greater than at the lower elevation.

These observations are substantiated by the present measurements (see Table 1 and fig. 9) where the total ultra-violet, for $m=1.2$, was u. v. $Q .=1,150$ microcalories at the 10,500 -foot elevation as compared with $u . v . Q .=1,080$ microcalories per $\mathrm{cm}^{2}$ per minute at the 7,250 -foot elevation, a difference of only about 8 per cent under average conditions at that season, September, 1929.

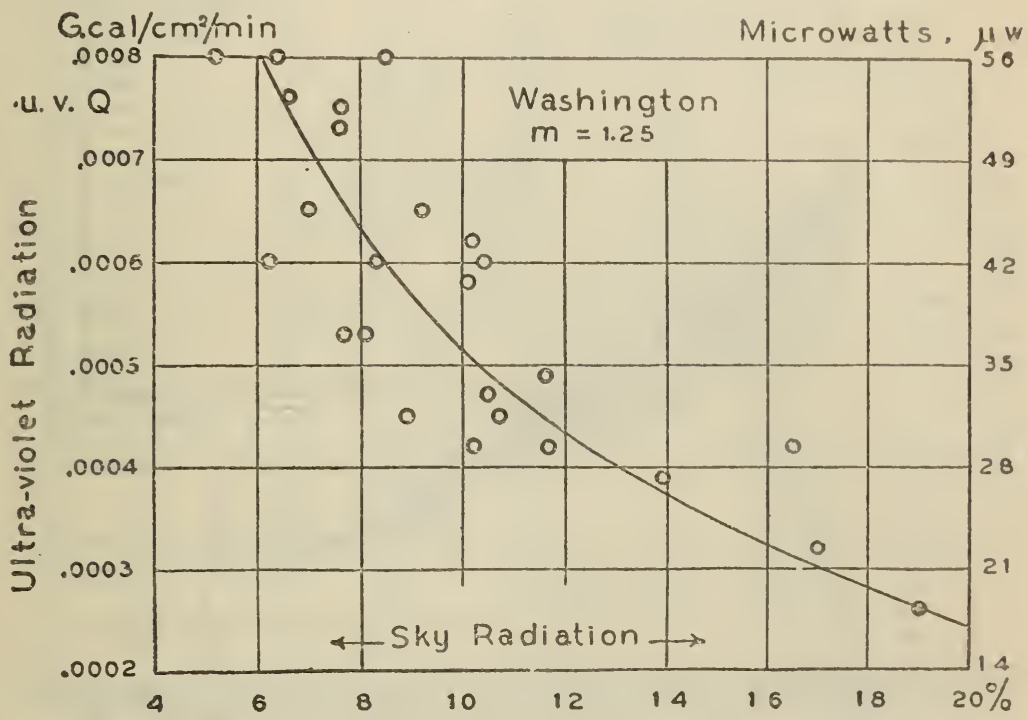

FIGURE 8.-Percentage of sky radiation present in the total ultra-violet of wave lengths less than $313 \mathrm{m \mu}$, at Washington, for air mass $m=1.25$. (See fig. \%.)

The greater erythemic effectiveness of the ultra-violet rays, observed at the higher elevation is therefore to be ascribed to the presence of a greater amount of short wave lengths (at 297 to $302 \mathrm{m \mu}$ ) ultra-violet radiation, as indicated by the lower transmission, $a / I_{\text {o }}$ observed through the barium flint glass filter.

\section{EFFECT OF HUMIDITY ON SCATTERING}

But little information is available regarding the amount of scattering caused by water vapor along the path of the incident radiation.

The Washington measurements (Table 2) show that on the clearest days (absence of smoke and dust) only about 5 per cent of the total $u$. v. $Q$. is scattered sky radiation, the average being about 10 per cent of the total, rising to 19 per cent on April 12, 1930, as a result of the Shenandoah forest fire. 
The most interesting results on the effect of humidity were obtained at the Arizona stations, where observations were begun near the close of the rainy season when the pressure of the aqueous vapor at the 7,250 -foot elevation was $10 \mathrm{~mm}$. This was followed by a period of low vapor pressure (4 to $5 \mathrm{~mm}$ ) which, fortunately for a verification of the earlier observations, was terminated by a day (Sept. 19, 1929, Table 1) of relatively high vapor pressure.

As shown in Figure 10, where the total ultra-violet radiation, $u$.v. Q., is plotted against the total solar radiation intensity, $Q$, during the period of relatively high vapor pressure, the ultra-violet component, $u . v . Q$., is distinctly higher for a given value of $Q$ (group $A$ ) than during the period of low vapor pressure (group $B$ ), so that with a solar intensity of $Q=1.3 \mathrm{~g}$ cal. the average $u . v$. $Q$. was as high

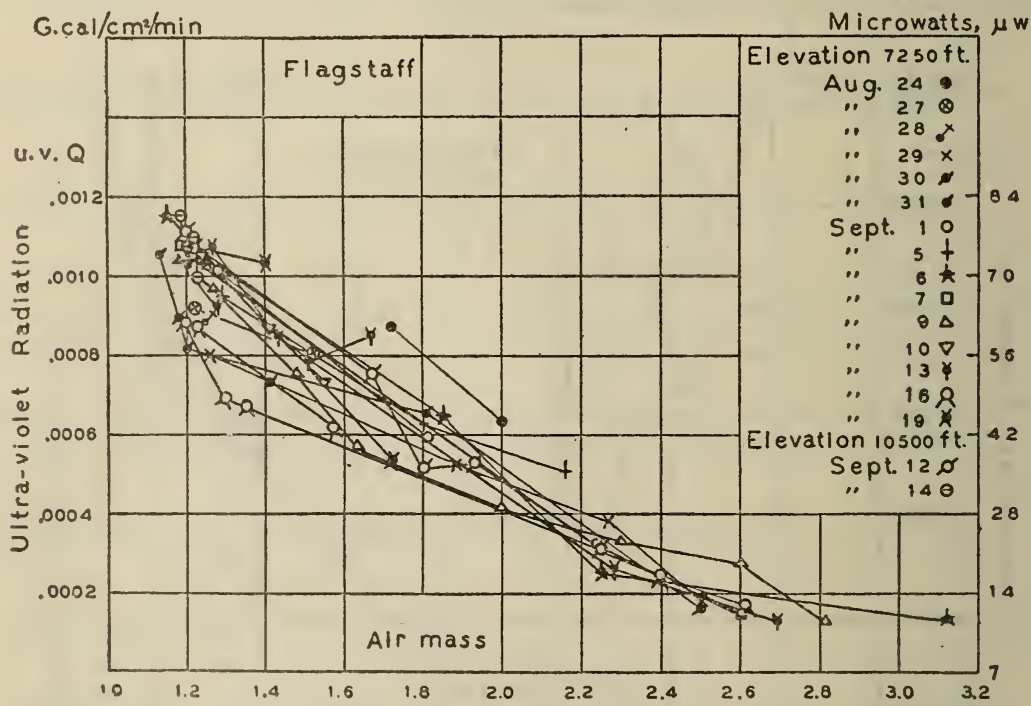

FIGURE 9.-Daily variation in the total ultra-violet radiation of wave lengths less than 313 mu, Flagstaff, Ariz., August and September, 1929

$(0.0009 \mathrm{~g}$ cal. $)$ as was observed at the low vapor pressures $(4 \mathrm{~mm})$ when $Q=1.5 \mathrm{~g}$ cal. It is to be noted, however, that, while the total ultra-violet component at the 7,250-foot elevation is almost as high as observed at the 10,500-foot elevation, the spectral quality is different, containing a somewhat less amount of the short-wave lengths 290 to $300 \mathrm{~m} \mu$. This conclusion follows from the proper interpretation of the transmissions observed with the barium flint glass screens.

It is relevant to add that the effect of humidity upon the total incident solar radiation, $Q$, is well known to meteorologists, the relatively lower values of $Q$ in June and July being caused, in part, by the increased amount of water vapor in the atmosphere.

In this connection it is interesting to note the observations of Dannmeyer (17), showing the importance of sky radiation upon the total biologically active ultra-violet incident in high latitudesIceland, Finland, Norway, etc. During a period of exceptionally clear weather, at midday, in June and July, the total ultra-violet in direct sunlight in Iceland (measured with a cadmium photo-electric 
cell) was never greater than 45 per cent of the $u . v$. $Q$ received from the whole sky. He comments upon the biological effect of this sky radiation upon the natives, living in towns surrounded by basalt walls $400 \mathrm{~m}$ high, whose faces, blanched during the long dark winter's, show a ruddy hue long before the sun shines directly into the fiords.

\section{DAILY VARIATION IN ULTRA-VIOLET RADIATION}

The daily variation in intensity of the ultra-violet component, $u$. v. Q., from an imperceptible amount at sunrise and sunset to a maximum at the noon hour is well known and needs but little comment in the present paper.

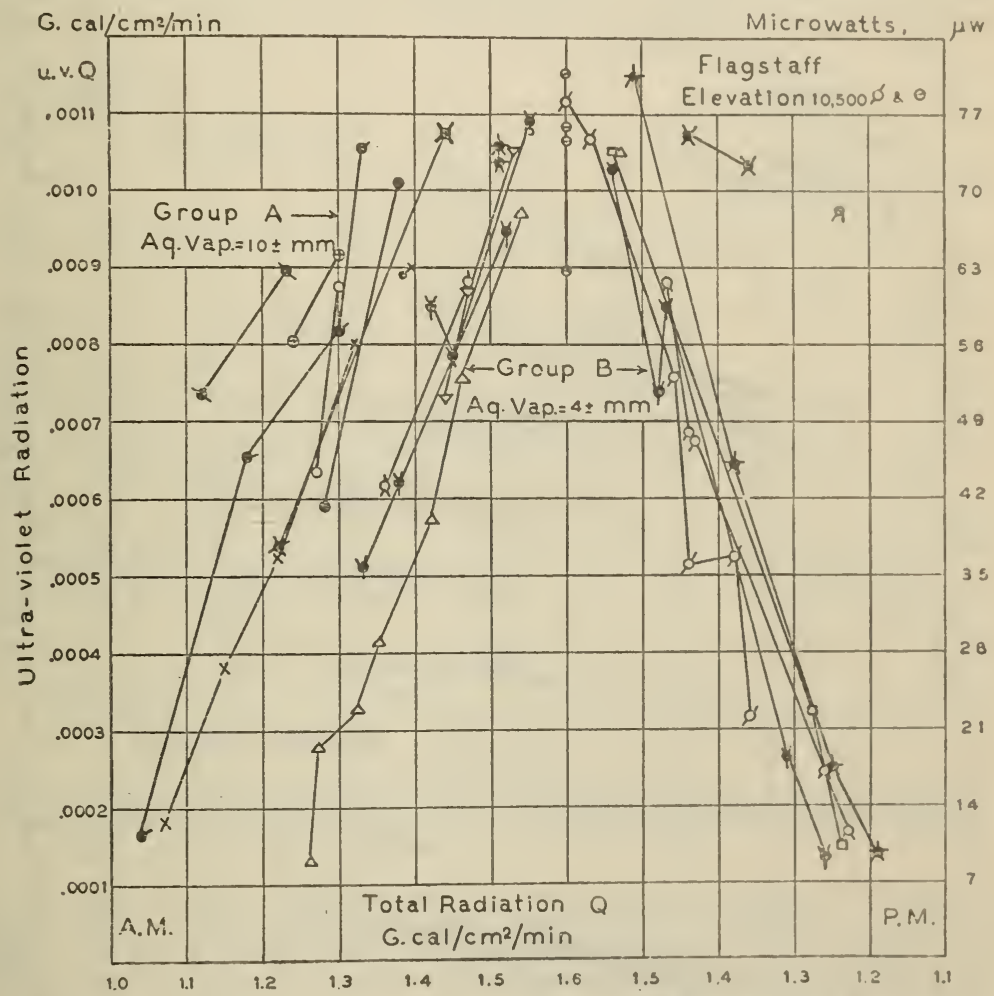

Figure 10.-Daily variation in ultra-violet radiation $(u, v, Q)$ relative to the total incident solar radiation $(Q)$ in $g$ cal. per $\mathrm{cm}^{2}$ per minute, at Flagstaff, Ariz., showing the effect of aqueous vapor

In Figure 10 are depicted some of the measurements of the $u . \imath$. Q. made at the Flagstaff, Ariz., stations. The Washington measurements (fig. 7), being plotted against air mass, do not distinguish between the forenoon and afternoon observations. However, in both cases, the wide variations in the $u . v . Q$., throughout the day and on different days, indicate the futility of attempting to specify intensities for average meteorological conditions. Fortunately, common experience shows that the human body is not greatly affected by these variations in intensity, so that a precise dosage meter is not necessary to evaluate the intensities in heliotherapy as now practiced. 


\section{SEASONAL VARIATION IN ULTRA-VIOLET RADIATION}

In Figure 11 are plotted the various observations of ultra-violet radiation, $u$. v. Q. at Washington, for different times of the day (different air masses given at the top of fig. 11) during different

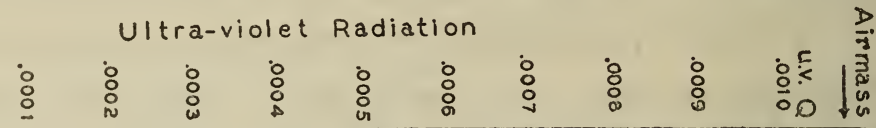

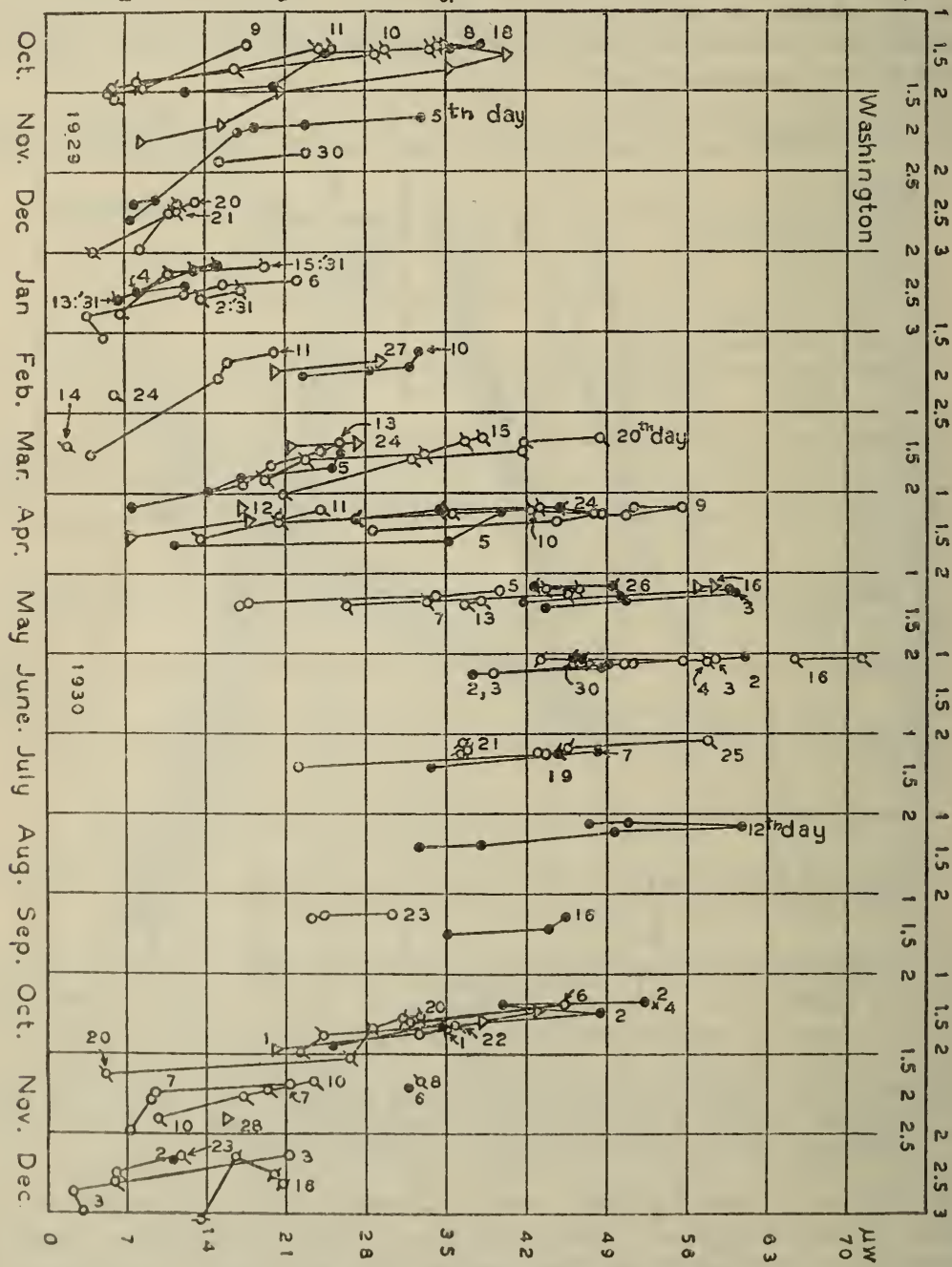

FIgURE 11.-Daily and seasonal variation in ultra-violet solar radiation of wave lengths less than $313 \mathrm{m \mu}$, in Washington, October, 1929, to December, 1930

months of the year. The envelope, drawn through the maximum and the minimum values, produces an interesting curve, showing, the well-known periodicity in the intensity, varying from a sharply defined minimum in December to a broadly defined maximum extend- 
ing from May to August. No attempt is made to correlate the incident ultra-violet with solar activity (sun spots) which is being investigated by Pettit (9).

In Figure 12 the data are plotted to illustrate the amount of ultraviolet incident at Washington at different hours of the day (usually the forenoon) during the year 1930 and the first part of 1931. The low average values in July are owing to haziness caused by the drought (dust) and forest fires.

As illustrated in Figure 12, the ultra-violet component radiation, u. v. Q. of biological importance in sunlight is imperceptibly small before $9 \mathrm{a} . \mathrm{m}$. (and after $3 \mathrm{p} . \mathrm{m}$.) in winter; and only during three to four hours, in the middle of the day does it become of real importance. However, by February, and especially by the beginning of March,

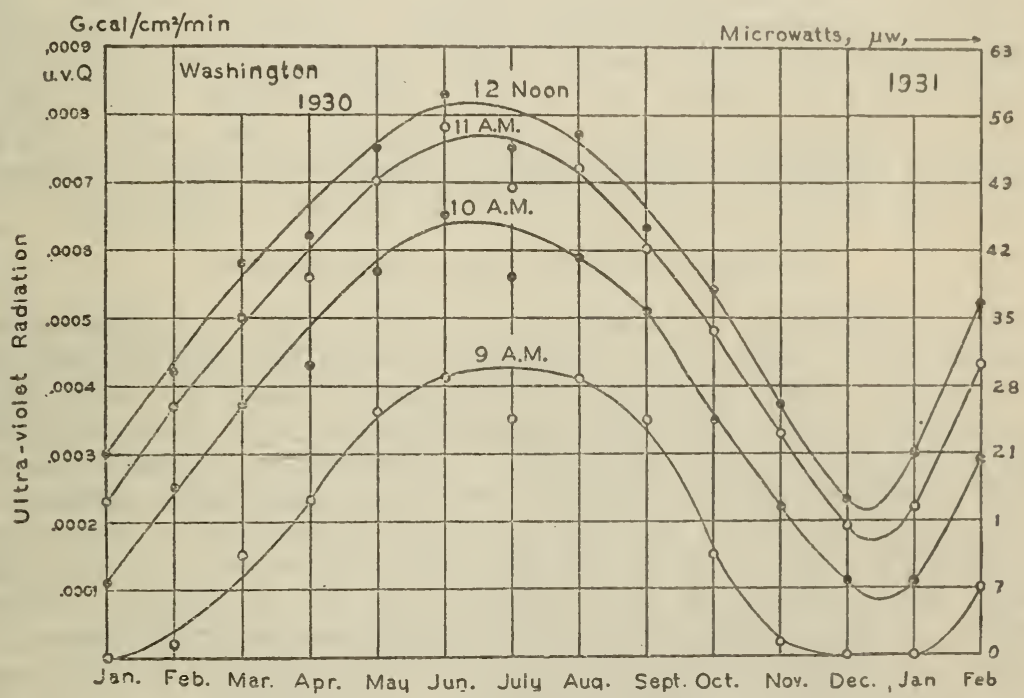

FIGURE 12.-Average ultra-violet solar radiation intensities of wave lengths less than $313 \mathrm{m \mu}$ at the even hours, 9 a. m. to noon on the clearest days throughout the year 1950, in Washington

the amount of ultra-violet is greatly increased. This is a point that appears to have been overlooked in some of the earlier biological tests which were made in February, and, hence, do not apply to conditions that obtain in December. For even if there are more clear days in December than in January and February, accepting the observations of Russell, Massengale, and Howard (11) that the duration of the effect of a single exposure to ultra-violet radiation upon bone formation in chicks lasts about two wecks, then the biological action obtained on a single clear day in January and February would be far more effective than several days of exposure to the sun, during December.

The foregoing data are interesting in showing that in addition to the daily variation in the intensity of the ultra-violet component, $u$. v. Q., there is the well-known seasonal variation which is a minimum at the winter solstice, December 22, when at the noon hour on the clearest day, in Washington, the $u . v$. Q. is less than 200 micro- 
calories (about $14 \mu \mathrm{w}$ ) per $\mathrm{cm}^{2}$ or about one-fourth the maximum values at the summer solstice. This value seems to be remarkably high, but it is to be noted that the total amount of ultra-violet, $u . v$. Q., observed in December is of wave lengths 305 to $313 \mathrm{~m} \mu$, especially of the latter wave lengths, which have a low biological action.

If we consider only the energy included under the rubescence curve, $R$, in Figure 2, which equates the spectral energy according to its biological (erythemic) effect, then the maximum biologically active ultra-violet component, $u$. v. Q. calculated from the average solar spectral energy curve, at the winter solstice, is about one-fifth to one-sixth the maximum at the summer solstice.

The biological tests of Tisdall and Brown (10) made at Toronto (latitude $43^{\circ} 6^{\prime}$ ), indicate that during the winter months the antirachitic effect is about one-eighth as great as in April and May. Assuming the same meteorological conditions at Toronto and at Washington, it is to be noted, however, that in making biological tests the animals must be exposed two to three hours per day, on successive days, for a period of a month, during which time the average $u_{.} v . Q$. especially during the winter months, is much lower than the average of only the clearest days, included in the present investigation.

\section{ULTRA-VIOLET IN SKY RADIATION}

It is well known that there is a greater amount of ultra-violet, relative to the total, in sky radiation than in the directly incident solar ways. Hence, although the intrinsic brilliancy of a small area of the sky is much lower than direct sunlight, since the area of the sky is 92,000 times that of the sun, the integrated amount of ultraviolet incident from the whole sky can be an appreciable amount.

On page 682 of the previous paper (2) measurements were cited indicating a value of $u . v . Q=0.00029 \mathrm{~g} \mathrm{cal} / \mathrm{em}^{2} / \mathrm{min}$. for practically the whole north sky on an exceptionally clear day in March. When reduced to the present system of measurement (using the factor, $F$, in fig. 5 , in order to obtain the value for wave lengths less than $313 \mathrm{~m} \mu$ ) this value amounts to (approximately) $u . v . Q=0.00006 \mathrm{~g} \mathrm{cal} / \mathrm{cm}^{2} / \mathrm{min}$. or about one-fifteenth of June sunlight, which is an appreciable amount.

By means of a uranium photo-electric cell, sensitive to wave lengths less than $334 \mathrm{~m} \mu$ and the barium flint glass screen, it was found that, of the total intensity measured in foot candles, the light from the north sky contains about 50 per cent more of the biologically active ultra-violet component than direct sunlight. However, since the intensity of skylight that can pass through an average sized window, and shine upon an object $5 \mathrm{~m}$ back of the window, is only 10 to 12 foot candles or about $1 / 800$ to $1 / 1000$ of full sunlight, it follows that it would be necessary to expose an object for 14 to 15 hours to receive the same amount of ultra-violet as would be obtained in a 1-minute exposure to direct June sunlight (12).

\section{COMPARISON OF DATA}

As already mentioned, practically all the quantitative data, heretofore available, on the biologically active ultra-violet in sunlight, were derived by calculation from an assumed average spectral energy curve $(9,13)$ which formed also the basis of one method of attack, previously 
used by the writers (2). In the present investigation the average solar spectral energy curve is used merely as a secondary factor for reducing to an absolute energy basis the ultra-violet energy measurements obtained under various atmospheric conditions. While the data obtained by these two methods of attacling the problem are in reasonably good agreement, nevertheless the present measurements show that such comparisons can be used only in a general discussion of the problem, and that in accurate, quantitative biological tests, simultaneous local ultra-violet measurements must bo made.

From his analysis of the solar spectral energy curve observed on Mount Wilson, supplemented by measurements with a silvered photoelectric cell that has a maximum response at about $320 \mathrm{~m} \mu$, Pettit (9) deduced that the intensity of the ultra-violet component of wave lengths 290 to $310 \mathrm{~m} \mu$, in midsummer sunlight amounts to about 70 microwatts $(\mu \mathrm{w})$ per $\mathrm{cm}^{2}$. This amounts to about $90 \mu \mathrm{w}$ per $\mathrm{cm}^{2}$ for wave lengths 290 to $313 \mathrm{~m} \mu$. The highest values observed in Washington (wave lengths 290 to $313 \mathrm{~m} \mu$ ) are close to $70 \mu \mathrm{w}(0.0010 \mathrm{~g}$ $\mathrm{cal} / \mathrm{min}$., fig. 7) per $\mathrm{cm}^{2}$.

The method of observation used by Grieder and Downs (5) has an element of similarity to the present method in that the per cent of the total incident energy that is located in the ultra-violet and visible spectrum, is determined by means of filter of red glass. It is therefore necessary to observe the spectral energy curve of only the ultraviolet and the visible part of the spectrum by means of a spectropyrheliometer (14) and, by graphical integration, determine the amount of radiation in the band of wave lengths 290 to $310 \mathrm{~m} \mu$.

The value of the ultra-violet component, observed by Greider and Downs (5) during the noon hour, at Springfield Lake, Ohio (altitude about 1,200 feet) ranges from $12 \mu \mathrm{w}$ per $\mathrm{cm}^{2}$ in October to $23 \mu \mathrm{w}$ per $\mathrm{cm}^{2}$ in June, or about 16 and $30 \mu \mathrm{w}$ per $\mathrm{cm}^{2}$, respectively, using the long wave length limit at $313 \mathrm{~m} \mu$. Deducting about 10 per cent from the herein-recorded measurements for sky radiation, the corresponding Washington measurements are approximately 35 and $50 \mu \mathrm{w}$ per $\mathrm{cm}^{2} 0.0005$ and $0.00073 \mathrm{~g}$ cal. (fig. 12), respectively. Their measurements at Cragmor, Colo. (altitude about 6,500 feet), gave $16 \mu \mathrm{w}$ per $\mathrm{cm}^{2}$ in November to $53 \mu \mathrm{w}$ per $\mathrm{cm}^{2}$ in June, or 21 and $69 \mu \mathrm{w}$ per $\mathrm{cm}^{2}$, respectively, using the long-wave length limit at $313 \mathrm{~m} \mu$. Extrapolating the herein-described measurements at Flagstaff, Ariz. (fig. 9), to air mass $m=1.02$ (June) and deducting 10 per cent for sky radiation gives $77 \mu \mathrm{w}$ per $\mathrm{cm}^{2}(0.0011 \mathrm{~g}$ cal.).

Supplementing these radiometric measurements are the physiological tests of Luckiesh (15) who, from determinations of minimum perceptible erythema and computations of energy between 280 and $310 \mathrm{~m} \mu$ produced by a tungsten-filament lamp with a special bulb, concludes that the best midsummer sunlight supplies not less than $25 \mu \mathrm{w}$ per $\mathrm{cm}^{2}$ with a probable value of $40 \mu \mathrm{w}$ per $\mathrm{cm}^{2}$ of ultra-violet energy shorter than $310 \mathrm{~m} \mu$, or 33 and $52 \mu \mathrm{w}$ per $\mathrm{cm}^{2}$, respectively, for wave lengths less than $313 \mathrm{~m} \mu$.

From the foregoing it appears that the present method of measuring ultra-violet solar radiation gives slightly higher values than the method employed by Greider and Downs, and lower values than obtained by Pettit. The various estimates of the value of ultra-violet solar radiation of wave lengths less than $313 \mathrm{~m} \mu$, for mid-latitude sea-level stations, during midday in midsummer, are as follows: Pettit, $90 \mu \mathrm{w}$; 
Greider and Downs, $33 \mu \mathrm{w}$ (corrected for sky radiation); Luckiesh, 33 to $52 \mu \mathrm{w}$; Coblentz and Stair $55 \mu \mathrm{w}$ per $\mathrm{cm}^{2}(20)$.

Considering the variety of methods of attack and of reducing the measurements, and the marked local differences in meteorological conditions at the different stations, these estimates are in as good agreement as can be expected. For, while this general agreement is of interest, the question of primary importance is a quick, reliable, and convenient method of determining, in absolute value, the amount of biologically effective ultra-violet radiation incident at a given station.

Heliotherapists find that the best time of the day to give sunbaths is in the early forenoon ( 8 to 9 a. m.) and late afternoon in summer, and during the noon hours in winter. From a correlation of their radiometric data with these biological data, Greider and Downs (5) estimate the dosage intensity of "clinical sunlight," used in heliotherapy amounts to about $14 \mu \mathrm{w}$ per $\mathrm{cm}^{2}$. On the basis of the present method of observation, which includes wave lengths less than $313 \mathrm{~m} \mu$ the dosage unit of "clinical sunshine" is about $20 \mu \mathrm{w}$ per $\mathrm{cm}^{2}$. This is considerably smaller than the estimate previously published $(8,2)$. However, the former estimate was based upon an assumed upper limit of $70 \mu \mathrm{w}$, which is negatived by the results of the present research (20).

\section{SUMMARY AND ACKNOWLEDGMENTS}

The present paper is a contribution to the investigation of instruments and methods of radiometry, in particular to the quantitative measurement of the component of ultra-violet solar radiation, of wave lengths less than $313 \mathrm{~m} \mu$, which biologists recognize as of especial importance in the prevention and cure of rickets.

The apparatus used consisted of a nonselective radiometer (a thermocouple) permanently covered with a cell of water and glass filter, Corning G986A, which combination is highly transparent to ultra-violet and largely opaque to the visible and the infra-red rays. By temporarily placing a screen of barium flint glass in the path of the incident rays, the ultra-violet of wave lengths less than $313 \mathrm{~m} \mu$ is evaluated by exclusion from the total ultra-violet measured.

Observations were made at Washington, D. C. (latitude $38.9^{\circ}$; altitude 350 feet) at the Lowell Observatory, Flagstaff, Ariz. (latitude $35.2^{\circ}$; altitude 7,250 feet) and on the San Francisco Peaks, near Flagstaff (altitude 10,500 feet).

Data are presented on the incident ultra-violet solar radiation as affected by $(a)$ atmospheric pollution, $(b)$ altitude, $(c)$ humidity, $(d)$ time of the day, $(e)$ season of the year, and $(f)$ sky radiation.

Among the outstanding items of interest is the great reduction in intensity of ultra-violet radiation by atmospheric pollution by dust, smoke, and the combustion products of automobiles. This produces a wide variation in the daily and seasonal observations, especially at sea-level stations.

At high elevations the spectral quality of the ultra-violet component is richer in the short wave lengths $(295$ to $305 \mathrm{~m} \mu)$ than at sea-level; but owing to atmospheric scattering, the total amount of ultraviolet of wave lengths less than $313 \mathrm{~m} \mu$, at sea-level on the clearest days, is almost as large as at high altitudes. 
Atmospheric water vapor is an important contributing factor in scattering ultra-violet radiation, that is most easily observed with changes in humidity at high altitudes.

The amount of ultra-violet solar radiation of wave length less than $313 \mathrm{~m} \mu$, previously estimated to be 0.3 per cent of the total incident radiation, was an upper limiting value which is now superseded by more complete measurements, made on the clearest days in Washington, indicating noon hour values ranging from about 14 microwatts (200 microcalories per minute) per $\mathrm{cm}^{2}$ in December to about 56 microwatts ( $\$ 00$ microcalories per minute) per $\mathrm{cm}^{2}$ in June (20).

Acknowledgements are due to the Lowell Observatory, in particular Dr. C. O. Lampland, for the facilities placed at our disposal, including transportation to the San Francisco Peaks; also to Mrs. W. W. Coblentz for assistance in maling the observations in Arizona, and to J. M. Hogue for assistance in making some of the latest observations in Washington. Acknowledgment is due to Dr. H. H. Kimball, of the United States Weather Bureau, for the calibration of the Angström pyrheliometer used at the Flagstaff stations and to I. F. Hand for the numerous data on air masses $(m)$ and solar intensities $(Q)$ supplied in connection with the Washington measurements of ultraviolet radiation.

\section{REFERENCES}

1. Instruments and Methods of Radiometry. I, W. W. Coblentz, B. S. Bull. No. 85,4 , p. $391 ; 1908$.

Instruments and Methods of Radiometry. No. 188,9 , p. $7 ; 1912$.

Various Modifications of Bismuth Silver Thermopiles, W. W. Coblentz, B. S. Bull. No. 229, 11, p. 131; 1914.

Studies of Instruments for Measuring Radiant Energy in Absolute Value; An Absolute Thermopile, W. W. Coblentz and W. B. Emerson, B. S. Bull. No. 261, 12, p. 503; 1916 .

Sensitivity and Magnetic Shielding Tests of Thomson Galvanometers for Use in Radiometry, W. W. Coblentz, B. S. Bull. No. 282, 13, p. 423; 1916.

Instruments and Methods of Radiometry. III; Selective Radiometers, W. W. Coblentz, B. S. Bull. No. 319, 14, p. $507 ; 1918$.

A New Spectropyrheliometer and Measurements of the Component Radiations from a Quartz Mercury Vapor Lamp and the Sun, W. W. Coblentz and H. Kahler, B. S. Sci. Paper No. 378, 16, p. 233; 1920.

The results of studies of instruments for stellar and planetary radionetry are described in B. S. Bull. No. 244, and in B. S. Sci. Papers Nos. 438, 460, and 553 .

Tests of a vacuum radiometer are given in B. S. Bull. No. 46, and of a portable vacuum thermopile in B. S. Sci. Paper No. 413.

Filter methods of radiometry, used in isolating and measuring wide bands of radiation, particularly in the ultra-violet, are described in B. S. Sci. Papers Nos. 330 and 539, and in B. S. Research Papers Nos. 6 and 113.

2. Data on Ultra-Violet Solar Radiation and the Solarization of Window Materials, W. W. Coblentz and R. Stair, B. S. Research Paper No. 113, 3, p. $629 ; 1929$.

3. Note on Filter Radiometry of Incandescent Lamps, D. C. Stockbarger, A. L. M. Dingee and L. Burns, J. Opt. Soc. Am. and Rev. Sci. Inst., 18, p. 53; 1929.

4. Fabry and Buisson, Astrophys, J., 54, p. 297; 1921. O'Brien, Phys. Rev., 36, p. 381; 1930; and personal communication.

5. Greider and Downs, Trans. Illum. Eng. Soc., 25, p. 378; 1930.

6. Hess and Weinstock, J. Am. Med. Assn., 80, p. 687; 1923; Proc. Soc. Expt1. Biol. and Med., 24, p. 759; 1927.

Hess and Anderson, J. Am. Med. Assoc., 89, p. 1222; 1927.

Sonne and Reckling, Hospitalstidend, 70, p. 399; 1927.

Maughan, Am. J. Physiol., 87, p. 381; 1928. 
7. Hausser and Vahle, Strahlentherapie, 13, p. 59; 1922.

Hausser, Strahlentherapie, 28, p. 25; 1928.

Luckiesh, Holladay and Taylor, J. Ópt. Soc. Am., 28, p. 423; 1930.

8. Coblentz, Trans. Illum. Eng. Soc., 25, p. 359; 1930. Med. J. and Record, 130, p. $691 ; 1929$.

9. Pettit, Trans. Nat. Tuberculosis Assn. (24th Ann. Meeting); 1928.

10. Tisdall and Brown, Am. J. Diseases of Children, 34, p. 721; 1927.

11. The Duration of the Effect of Ultra-Violet Radiation in Chickens, Russell, Massengale and Howard, J. Biol. Chem., 80, p. 155; 1928.

12. The Biologically Active Component of Ultra-Violet in Sunlight and Daylight, W. W. Coblentz, Trans. Illum. Eng. Soc., 27, May-June; 1931.

13. Forsythe and Christison, Gen. Electric Rev., 32, p. 662; 1929.

14. Coblentz and Kahler, B. S. Sci. Papers No. 378, 16, p. 233; 1920.

15. Artificial Sunlight, by M. Luckiesh, D. van Nostrand Co., New York; 1930.

16. Annual Variation of Ultra-Violet in Sunlight, C. Dorno, Strahlentherapie, 31, p. 341; 1929.

17. Ultra-violet Sky Radiation in High Latitudes (Iceland), F. Dannmeyer, Strahlentherapie, 35, p. 607; 1930.

18. Instruments for Measuring Ultra-Violet Radiation and the Unit of Dosage in Ultra-Violet Therapy, W. W. Coblentz, Medical J. and Record, 130, p. 693; 1929. Brit. J. Radiology, 3, p. 354; 1930.

19. The effect of the Angular Opening of an Actinometer on the Measurement of Solar and Sky Radiation is discussed in a recent paper by F. Linke, Strahlentherapie, 39, p. 351; 1931.

20. A further study of the transmissions of the filters and correction factors indicates that the hereinrecorded values should be increased by, perhaps, 10 to 20 per cent.

Washington, February, 21, 1931. 